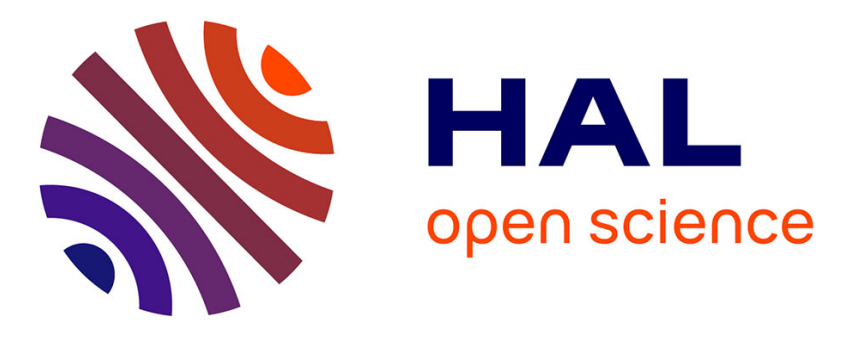

\title{
Acceleration of radiation belts electrons by oblique chorus waves
}

D Mourenas, A Artemyev, O Agapitov, V Krasnoselskikh

\section{To cite this version:}

D Mourenas, A Artemyev, O Agapitov, V Krasnoselskikh. Acceleration of radiation belts electrons by oblique chorus waves. Journal of Geophysical Research Space Physics, 2012, 117, A10212 (14 p.). 10.1029/2012JA018041 . insu-01179807

\section{HAL Id: insu-01179807 https://hal-insu.archives-ouvertes.fr/insu-01179807}

Submitted on 23 Jul 2015

HAL is a multi-disciplinary open access archive for the deposit and dissemination of scientific research documents, whether they are published or not. The documents may come from teaching and research institutions in France or abroad, or from public or private research centers.
L'archive ouverte pluridisciplinaire $\mathbf{H A L}$, est destinée au dépôt et à la diffusion de documents scientifiques de niveau recherche, publiés ou non, émanant des établissements d'enseignement et de recherche français ou étrangers, des laboratoires publics ou privés. 


\title{
Acceleration of radiation belts electrons by oblique chorus waves
}

\author{
D. Mourenas, ${ }^{1}$ A. Artemyev, ${ }^{2,3}$ O. Agapitov, ${ }^{2,4,5}$ and V. Krasnoselskikh ${ }^{2}$ \\ Received 18 June 2012; revised 20 August 2012; accepted 7 September 2012; published 10 October 2012.
}

[1] The redistribution of energy during the recovery phase of geomagnetic storms related to the acceleration of electrons in the Earth's outer radiation belt by cyclotron-resonant chorus waves is an important and challenging topic of magnetospheric plasma physics. An approximate analytical formulation of energy diffusion coefficients is derived in this paper, on the basis of a quasi-linear formalism valid for large enough bandwidths or for successive random scatter by uncorrelated waves of different frequencies and moderate average amplitudes. We make use of chorus wave parameterizations derived from CLUSTER measurements to show that oblique whistler waves can significantly increase the energy diffusion rate of small pitch angle electrons on the dayside. On the other hand, the energization rate of the more numerous high pitch angle electrons is typically reduced by a factor of 2 on the dayside, while it remains nearly unchanged on the nightside where high-intensity waves are less oblique. Besides, lifetimes are strongly reduced on the dayside, which could also impact the long-term time-integrated acceleration rates of injected electrons. Comparison between the analytical formulas and full numerical results demonstrates a good agreement and provides new scaling laws as a function of whistler mean frequency, plasma density and particle energy. It is also suggested that the enhancement of energy diffusion of low energy electrons $(<100 \mathrm{keV})$ at small pitch angles with oblique waves could result in an intensification of wave growth at latitudes higher than $15^{\circ}$. This might contribute to explain high chorus intensities measured by CLUSTER on the dayside at high latitudes.

Citation: Mourenas, D., A. Artemyev, O. Agapitov, and V. Krasnoselskikh (2012), Acceleration of radiation belts electrons by oblique chorus waves, J. Geophys. Res., 117, A10212, doi:10.1029/2012JA018041.

\section{Introduction}

[2] Energetic electrons trapped in the Earth's radiation belts are continuously scattered in pitch angle and energy by resonant interactions with whistler waves [Andronov and Trakhtengerts, 1964; Kennel and Petschek, 1966]. These interactions therefore represent an important component of the belt dynamics. Quasi-linear diffusion coefficients have been derived to quantify the corresponding loss and energization rates [Lyons et al., 1971, 1972; Lyons, 1974] and have recently been proved adequate for ensembles of uncorrelated waves of not-too-large amplitudes [Albert, 2010; Tao et al., 2011]. The outer radiation belt is highly variable, due to ever-changing levels of convection and variability of waves intensities with changing activity [Meredith et al., 2001; Lyons et al., 2005]. In particular, lower-band chorus waves excited by the anisotropy of the injected plasma sheet

\footnotetext{
${ }^{1}$ CEA, DAM, DIF, Arpajon, France.

${ }^{2}$ LPC2E, CNRS, University of Orleans, Orleans, France.

${ }^{3}$ Space Research Institute, RAS, Moscow, Russia.

${ }^{4}$ Physics Department, National Taras Shevchenko University of Kiev, Kiev, Ukraine.

${ }^{5}$ Le Studium, Institute for Advanced Studies, Orleans, France.

Corresponding author: D. Mourenas, CEA, DAM, DIF, FR-91297 Arpajon, France. (didier.mourenas@cea.fr)

C2012. American Geophysical Union. All Rights Reserved. 0148-0227/12/2012JA018041
}

electrons can lead to fast scattering of electrons into the loss cone on the dayside, on timescales ranging from hours to days during periods of storm-enhanced convection [Horne et al., 2005; Li et al., 2007]. A population of electrons with energy $<100 \mathrm{keV}$ can then transfer its energy to the waves and, later on, a small subpopulation of these electrons can get accelerated via their resonant interaction with the same waves of larger amplitudes [Horne et al., 2005; Thorne et al., 2005]. Since such energetic "killer" electrons in the $\mathrm{MeV}$ range are known to be very dangerous for satellite electronic equipments [Iucci et al., 2005], multidimensional "space weather" codes have been developed to model the spatio-temporal evolution of their flux [Barker et al., 2005; Shprits et al., 2008; Varotsou et al., 2008; Xiao et al., 2009; Fok et al., 2011]. Although radial diffusion is also known to play a significant role alike in the heating of electrons and the redistribution of energy [Ukhorskiy et al., 2011], it is important to explore the effects of the recently measured wavenormal angle distributions of chorus [Agapitov et al., 2011] on acceleration rates. In particular, all the previous studies have only considered parallel or slightly oblique chorus waves with $\theta<45^{\circ}$ [Roth et al., 1999; Summers et al., 2002; Horne et al., 2005; Li et al., 2007; Ni et al., 2008; Shprits et al., 2009], with the exception of $\mathrm{Ni}$ et al. [2011] which accounted for wave-normal angles $\theta$ up to $58^{\circ}$ in some of their simulations. While all the latter distributions were adequate in the vicinity of the equator, recent statistics of 
CLUSTER observations have demonstrated that most of the wave power at latitudes higher than $15^{\circ}$ corresponds to higher wave-normal angles $\theta \sim 45^{\circ}$ up to nearly $90^{\circ}$ [Agapitov et al., 2011; Artemyev et al., 2012b; O. Agapitov et al., A first approach to the statistical model of chorus waves activity in the inner magnetosphere, unpublished manuscript, 2012]. Clearly, taking into account a realistic wave-normal angle distribution is a necessary step for a better understanding of energization and precipitation processes in the outer belt. The MLT distribution of microburst precipitations is consistent with the MLT distribution of high-latitude waves which can scatter electrons into the loss cone in the frame of the quasi-linear theory [Thorne et al., 2005]. Trapping by very high amplitude oblique chorus waves may also possibly account for microburst precipitations [Kersten et al., 2011], while strong pitch angle diffusion toward the loss cone by oblique waves of weaker intensity but much longer duration may explain some features of pulsating auroras [Nishimura et al., 2010; Mourenas et al., 2012]. However, the effects of very oblique waves on the steady acceleration of electrons in the $\mathrm{MeV}$ range observed during the recovery phase of storms [Horne et al., 2005] have yet to be examined in details. The main goal of the present paper is precisely to investigate these effects, by means of both full numerical calculations of diffusion rates and their approximate analytical modeling. While not exact, an approximate analytical model can help to shed some light on the complicated variations of the diffusion rates as a function of the different parameters; it can also provide scaling laws and allow for quick estimations of the related timescales.

[3] In this paper, we consider only lower-band $\left(\Omega_{L H} \ll\right.$ $\left.\omega<\Omega_{c} / 2, \Omega_{L H}=\sqrt{\Omega_{c} \Omega_{c i}}\right)$ chorus whistler mode waves generated close to the geomagnetic equator [Burton and Holzer, 1974; Tsurutani and Smith, 1977; Trakhtengerts, 1999; Pokhotelov et al., 2008; Agapitov et al., 2010], where $\omega$ is the wave frequency, and $\Omega_{c i}, \Omega_{c}$ and $\Omega_{p e}$ are the local ion gyrofrequency, electron gyrofrequency, and plasma frequency, respectively. We consider the high-density limit $\Omega_{p e}^{2} \gg \Omega_{c} \omega$ introduced by Lyons [1974]. The high-density approximation of the quasi-longitudinal dispersion relation has been shown to apply relatively well to quasi-parallel lower-band chorus for equatorial ratio $\Omega_{p e} / \Omega_{c}>2.5[$ Albert, 2005; Glauert and Horne, 2005] and we shall discuss below its conditions of applicability for oblique waves.

[4] In the next section, analytical expressions for the energy diffusion coefficients are derived after performing both an approximate wave-normal angle averaging, a rough bounceaverage and a sum over the $n$-resonances, finally yielding analytical estimates of the energization rates of the trapped electrons. We follow the same method as in our previous papers [Mourenas and Ripoll, 2012; Mourenas et al., 2012], which were devoted solely to estimating pitch angle diffusion rates. To obtain estimations of energy diffusion coefficients $D_{E E}$ we derive analytical expressions for pitch angle diffusion coefficients $D_{\alpha \alpha}$ and use the relation between $D_{\alpha \alpha}$ and $D_{E E}$ obtained by Lyons [1974]. Scaling laws as a function of ambient density, geomagnetic field amplitude and central chorus frequency are also found. In the third section, the analytical model is compared to energy diffusion rates obtained from numerical computations of the diffusion coefficients using Glauert and Horne [2005] expressions together with realistic wave-normal angle distributions [Artemyev et al., 2012a]. The effects of a realistic latitudinal distribution of wave intensity [Artemyev et al., 2012b] are examined on the dayside as well as on the nightside and implications for electron acceleration/deceleration in the outer belt are also briefly discussed.

\section{Analytical Diffusion Coefficients and Timescales for Energy Diffusion}

[5] The waves spectral density is assumed to be Gaussianshaped $B_{\text {wave }}^{2}(\omega) \sim \exp \left(-\left(\omega-\omega_{m}\right)^{2} / \Delta \omega^{2}\right)$ with mean frequency $\omega_{m}$ and variance $\Delta \omega \sim \omega_{m} / 2$, while its distribution with wave-normal angle $\theta$ is described by a function $g(\theta)$. For outer-belt chorus, CLUSTER statistics [Artemyev et al., 2012a; Agapitov et al., unpublished manuscript, 2012] show that one can take $g(\theta)$ non-null and constant for $\theta$ lying between the Gendrin angle $\theta_{g}=\arccos \left(2 \omega_{m} / \Omega_{c}\right)$ and the resonance cone angle $\theta_{r}=\arccos \left(\omega_{m} / \Omega_{c}\right)$ at latitudes $\lambda>15^{\circ}$, with a roughly constant non-null portion at lower $\theta<\min$ $\left(\theta_{g}, \pi / 4\right)$ at $\lambda<15^{\circ}$ [see also Mourenas et al., 2012]. The plasma trough density model of Sheeley et al. [2001] can be used to model the electron density, leading to $N_{e} \sim 100(3 /$ $L)^{4} \mathrm{~cm}^{-3}$ on the day-side, consistent with saturated flux tube volume increasing as $L^{4}$ [Angerami and Carpenter, 1966]. We further assume that the plasma density does not vary significantly along the field line as long as latitude remains lower than $35^{\circ}$. This corresponds to recent studies [Denton et al., 2006] showing that density varies by less than a factor 1.5 over this range of latitudes at $L=5$ to 7 , and only slightly more at $L=4$ to 5 .

[6] The local pitch angle quasi-linear diffusion coefficient $D_{\alpha \alpha}(\alpha)$ of Lyons [1974] has been conveniently rewritten by Albert [2007] in the form of a weighted-average between $\theta_{\text {Min }}$ and $\theta_{\text {Max }}$, so that $D_{\alpha \alpha} \approx\left\langle D_{\alpha \alpha}\right\rangle_{\theta}$. The present approach builds on the above mentioned weighted-average formulation, but it is performed slightly differently to account for the variations of the averaged function with $\theta$. We perform integration over $\theta$-angle taking into account the dependence of $D_{\alpha \alpha}$ on $\theta$ (see details in Mourenas et al. [2012, Appendices C and D]), while Albert [2007] used the mean value theorem to estimate $D_{\alpha \alpha}(\alpha)$ by evaluating it at a wellchosen $\theta_{0}$. The latter approach is actually reasonable in the small- $\theta$ range, where one can safely neglect variations of $\theta$ (e.g., Summers et al. [2007] used $\theta_{0}=0$ for $D_{\alpha \alpha}(\alpha)$ estimates under the assumption of parallel wave propagation).

[7] In order to calculate the averaged pitch angle diffusion rate one needs to evaluate the local resonant pitch angle $\alpha_{R}$ as a function of equatorial value $\alpha_{0}$ and latitude $\lambda_{R}$, where resonant condition is satisfied. Let us now consider the cyclotron resonances, which are dominant at high enough energy, i.e., typically $E>100 \mathrm{keV}$ for chorus waves.

[8] For lower-band $\left(\omega_{m} / \Omega_{c}<1 / 2\right)$ chorus waves, which are right-hand polarized oblique whistler mode waves, the Appleton-Hartree dispersion relation valid for $\omega_{m}^{2} \gg$ $\omega_{L H}^{2}$ [Stix, 1962] can be reduced to the simplified dispersion relation $(c k)^{2} \approx \Omega_{p e}^{2} \omega /\left(\Omega_{c} \cos \theta-\omega\right)$ [Helliwell, 1965; Lyons, 1974] provided that two conditions are satisfied along field lines: $\Omega_{p e}^{2} /\left(\Omega_{c} \omega\right) \gg 1$ and $\sin \theta\left(\Omega_{c} /\right.$ $\left.\Omega_{c 0}\right)\left(\Omega_{c 0} / \Omega_{p e}\right)<\left(\Omega_{c} \cos \theta / \omega_{m}\right)^{1 / 2}$, where $\Omega_{c 0}$ is equatorial 
value of $\Omega_{c}$. It can also be shown that the maximum latitude $\lambda_{M M}$ where resonance exists over the large- $\theta$ range is reached near the loss cone edge. It is estimated in Appendix A from the simplified dispersion and adiabatic invariance as $\lambda_{M M} \leq 35^{\circ}$ for $L=4$ to 6 . When $\Omega_{p e} / \Omega_{c 0}>2.5$, the second inequality written just above determines the range of validity of the simplified dispersion. For consistency, the simplified dispersion and the ensuing analytical estimates can then be used only for $\Omega_{p e} / \Omega_{c 0} \geq 4$ for $\cos \theta$ comprised between $\cos \theta_{g}$ and $1.3 \cos \theta_{r}$. At lower density, the full Appleton-Hartree dispersion relation should be used, making analytical calculations practically untractable. In the opposite limit of moderately oblique waves (on the nightside), the simplified dispersion relation applies in the high-density limit $\left(\Omega_{p e} / \Omega_{c 0}\right)^{2} \gg\left(\omega_{m} / \Omega_{c 0}\right)\left(\Omega_{c} / \Omega_{c 0}\right)$. Then, resonance latitudes are smaller than $\lambda_{\operatorname{MAX}} \sim 30^{\circ}$ at pitch angles $\alpha_{0}>30^{\circ}$ for energies $E \geq 0.1 \mathrm{MeV}$ [Mourenas et al., 2012]. This leads roughly to a condition $\Omega_{p e} / \Omega_{c 0}>2.5$, in rough agreement with numerical simulations from Glauert and Horne [2005] in the case of quasi-parallel chorus waves.

[9] Now, let us consider the parameter range $\Omega_{p e} / \Omega_{c 0} \geq 4$ where the simplified dispersion should be approximately valid. Using the simplified dispersion (instead of the Appleton-Hartree one) will of course induce some errors in the determination of the resonance frequency at a given latitude. For moderately oblique waves interacting with electrons of pitch angle $\alpha_{0}>30^{\circ}$ and energy $E \geq 0.1 \mathrm{MeV}$ at relatively moderate latitudes, this discrepancy should remain negligible. On the other hand, for very oblique waves at high latitudes, the local error can increase sensibly. At high latitudes, however, the error in the value of the resonance frequency will most often correspond to a small shift of the latitude at which resonance actually occurs near peak wave power (due to the very rapid increase of $\Omega_{c}$ with latitude). In general, this should not induce large errors in the bounceaveraged diffusion rates after integration over latitudes.

[10] In order to estimate analytically the bounce-averaged diffusion rates, we make the following approximations: First, we estimate the latitudinal range where resonance occurs (for given electron energy and pitch angle) mainly from resonance condition and adiabatic invariance, taking into account that $\omega$ can vary between $\omega_{m}-\Delta \omega$ and $\omega_{m}+\Delta \omega$. This yields a latitudinal range of resonance $\Delta \lambda$. As this latitudinal range is relatively narrow $\left(\Delta \lambda<35^{\circ}\right)$ and since the variation with latitude of the integrand in the bounce integral remains weak over the main part of this latitude range (over the whole latitude range for large pitch angles $\alpha_{0}>30^{\circ}$ [see Mourenas et al., 2012, Appendix D]; at smaller pitch angles, over the most important part of $\Delta \lambda$ where the integrand is largest, as shown in Appendix A), we then assume that the diffusion rate integrated over $\Delta \lambda$ can be estimated as the integrand of the bounce integral taken at an average latitude $\langle\lambda\rangle$ and at $\omega=\omega_{m}$, multiplied by $\Delta \lambda$ and by $\left\langle B_{\text {wave }}^{2}\right\rangle_{\omega} / B_{\text {wave }}^{2}\left(\omega_{m}\right)$ to take into account the average wave power [Mourenas et al., 2012; Mourenas and Ripoll, 2012].

[11] The above approximations should not yield large errors in the bounce-averaged diffusion rates, provided that $\Delta \lambda$ is small enough for integrand variations to remain small and for possibly significant local errors (due for instance to variations in local resonant frequency) to more or less compensate each other when averaged over this finite latitudinal range. Clearly, this approach should be more appropriate for relatively narrow Gaussian chorus spectra with $\Delta \omega \sim \omega_{m} / 2$ and cutoffs at $\left|\omega-\omega_{m}\right|=\Delta \omega$. While this is representative of typical chorus spectra recorded on the dayside, nightside spectra have often been taken as twice wider with cutoffs at $\left|\omega-\omega_{m}\right|=2 \Delta \omega$ [Li et al., 2007]. In the present paper, we shall use only the first expression of the cutoffs, because our analytical developments are more adequate in this case. While this could lead to some differences with true nightside diffusion coefficients, a large portion of the neglected part of the nightside spectra corresponds to wave power less than $1 / 7$ of the peak power, which is quite small.

[12] To calculate the resonant pitch angle $\alpha_{R}$ for a given electron momentum $p=\sqrt{\gamma^{2}-1}$ (where $\gamma$ is the relativistic factor and $p$ is normalized on $m_{e} c$ ) we combine the simplified dispersion relation with resonant condition $\gamma \omega_{m}-p k \cos \theta \cos \alpha_{R}=-n \Omega_{c}$. From these two equations we exclude wave number $k$ and obtain an expression for $\alpha_{R}$

$$
\cos \alpha_{R}=\frac{\left|n-\frac{\gamma \omega_{m}}{\Omega_{c}}\right|\left|1-\frac{\omega_{m}}{\Omega_{c} \cos \theta}\right|^{1 / 2}}{p \varepsilon_{m} \sqrt{\cos \theta}}
$$

where $\varepsilon_{m}=\sqrt{\omega_{m} / \Omega_{c}}\left(\Omega_{p e} / \Omega_{c}\right)$. Although for large $L$ shells it can be important to consider the effects of a more realistic, non-dipolar magnetic field [see, e.g., Orlova and Shprits, 2010], we assume here for simplicity a dipole model $B=B_{0} \sqrt{1+3 \sin ^{2} \lambda} / \cos ^{6} \lambda$ with magnetic latitude $\lambda$ and equatorial magnetic field value $B_{0}$ for $L<6$. Combining with the equation for $\alpha_{R}$ and considering adiabatic motion along a bounce-trajectory (i.e. $\sin ^{2} \alpha / B=\sin ^{2} \alpha_{0} / B_{0}$ ) leads to the relation

$$
\begin{aligned}
\cos \alpha_{R} & =\frac{\sin ^{3} \alpha_{R}}{\sin ^{3} \alpha_{0}} \frac{\left|n-\frac{\gamma \omega_{m}}{\Omega_{c}}\right|\left|1-\frac{\omega_{m}}{\Omega_{c} \cos \theta}\right|^{1 / 2}}{p \varepsilon_{m 0} \sqrt{\cos \theta}} \\
& =\frac{\left|n-\frac{\gamma \omega_{m}}{\Omega_{c}}\right|\left|1-\frac{\omega_{m}}{\Omega_{c} \cos \theta}\right|^{1 / 2}\left(1+3 \sin ^{2} \lambda\right)^{3 / 4}}{p \varepsilon_{m 0} \sqrt{\cos \theta} \cos ^{9} \lambda}
\end{aligned}
$$

[13] Throughout the present paper, the variables with subscript "0" represent equatorial values.

[14] The large- $\theta$ part of the wave-normal angle distribution, such that $\theta \geq \max \left(\pi / 4, \theta_{g}\right)$, has already been shown by [Mourenas et al., 2012] to contribute the most to diffusion rates, at least for the dayside realistic chorus distribution considered here [Artemyev et al., 2012b]. Thus, we shall first assume that this large- $\theta$ part represents the major contribution to diffusion on the dayside when oblique waves are included. The nightside chorus distribution consisting of less oblique waves [Li et al., 2011; Artemyev et al., 2012b] will be considered later, at the end of this section.

[15] On the dayside, resonant diffusion then comes from resonant $\theta_{R}$-values lying between the Gendrin angle $\theta_{g}$ and the resonance angle $\theta_{r}$ [Gendrin, 1961]. Such very oblique chorus waves are commonly observed in the outer belt [Santolik et al., 2009; Haque et al., 2010, 2011; Agapitov et al., 2010, 2011; Li et al., 2011]. At the Gendrin angle, the waves remain guided along the geomagnetic field line as they propagate. However, density and magnetic field gradients from low to high latitude usually refract the waves, 
increasing their wave-normal angle up to the resonance cone, potentially leading to reflection at lower altitude [Shklyar et al., 2004; Chum and Santolik, 2005].

[16] The wave-normal angle integral in the Lyons et al. [1971] quasi-linear diffusion coefficients can be performed by splitting the integral into two parts. Diffusion coefficient for $n$th harmonic $D_{n}$ depends on Bessel functions $J_{n}(x)$, $J_{n \pm 1}(x)$ with argument $x=\left(c k p / \Omega_{c}\right) \sin \theta \sin \alpha$ [Lyons et al., 1971]. Using the resonant condition without term $\sim \omega_{m}$ for non-null $|n|$ this argument can be rewritten as $x=n \tan \alpha \tan \theta$. The first maximum of Bessel function corresponds to a certain value $x_{M}$ and we expand these functions for $x<x_{M}$ and $x>x_{M}$ in agreement with Mourenas and Ripoll [2012]. Therefore, the upper $D^{+}$and lower $D^{-}$parts of diffusion coefficient correspond, respectively, to the $x>x_{M}$ and $x<x_{M}$ limits in the Bessel functions classic expansions. In the pitch angle range $\alpha_{0}>\arcsin \left(\omega_{m} / \Omega_{c 0}\right) \sim 20^{\circ}$ corresponding roughly to $x>x_{M}$, it leads to [Mourenas et al., 2012]:

$$
\begin{aligned}
\left\langle\frac{D_{\alpha \alpha, \pm n}^{+}}{p^{2}}\right\rangle_{\theta_{g}}^{\theta_{r}} \approx & \frac{B_{w}^{2}}{B^{2}} \frac{\omega_{m} \Omega_{c}\left(1+\omega_{m}^{2} / \Omega_{c 0}^{2}\right)}{8 \gamma \Delta \omega p^{2} \varepsilon_{m}^{2} \cos \alpha \sin \alpha} \\
& \times \frac{\tan \theta_{r}\left(3+\tan ^{2} \theta_{r}\right)-\tan \theta_{g}\left(3+\tan ^{2} \theta_{g}\right)}{\left(1+\tan ^{2} \theta_{r}\right)^{3 / 2}-\left(1+\tan ^{2} \theta_{g}\right)^{3 / 2}}
\end{aligned}
$$

where $B_{w}$ is wave mean amplitude

$$
\int^{\left|\omega-\omega_{m}\right|<\Delta \omega} B_{\text {wave }}^{2}(\omega) d \omega=B_{w}^{2}
$$

[17] Note that local pitch angle diffusion rates (3) are independent of $n$. Therefore, many resonances can contribute. However pitch angle $\alpha_{R}$ is limited by condition (2) with $\cos \alpha_{R}<\sin \theta_{r}$ [see Mourenas et al., 2012, Appendix B], showing that, at a given latitude, $\theta_{R}\left(\lambda_{R}\right)$ can take any value smaller than $\theta_{r}\left(\lambda_{R}\right)$ only if $|n|<2 p \cos \alpha_{0} \Omega_{p e}\left(\omega_{m}+\Delta \omega\right) /$ $\Omega_{c 0}^{2}+\gamma\left(\omega_{m}+\Delta \omega\right) / \Omega_{c 0}$. At larger $n$-values, $\theta_{R}\left(\lambda_{R}\right)$ tends toward $\theta_{r}\left(\lambda_{R}\right)$ so that the integration range shrinks as $\left(\cos \theta_{r}\left(\lambda_{R}\right)-\cos \theta_{R}\left(\lambda_{R}\right)\right) / \cos \theta_{r}\left(\lambda_{R}\right) \propto 1 / n^{2}$, leading in equation (3) to $D_{n}$ decreasing rapidly like $1 / n^{2}$ at large $n$ [Mourenas et al., 2012]. In the opposite small pitch angle range $\alpha_{0}<\arcsin \left(\omega_{m} / \Omega_{c 0}\right)$ corresponding roughly to $x<x_{M}$, integration of $J_{|n|-1}^{2} \tan \theta \sqrt{1+\tan ^{2} \theta}$ leads to (cf. Appendix A):

$$
\begin{aligned}
\left\langle\frac{D_{\alpha \alpha, \pm n}^{-}}{p^{2}}\right\rangle_{\theta_{g}}^{\theta_{r}} \approx & \frac{B_{w}^{2}}{B^{2}} \frac{3 \Omega_{c} \omega_{m}\left(1+\omega_{m}^{2} / \Omega_{c 0}^{2}\right)(\tan \alpha)^{2|n|-2}}{16\left(1+|n|^{-1}\right) \gamma \Delta \omega p^{2} \varepsilon_{m}^{2} \cos ^{2} \alpha} \\
& \times \frac{\tan ^{2|n|} \theta_{r}\left(1+|n|^{-1}+\tan ^{2} \theta_{r}\right)}{\left(1+\tan ^{2} \theta_{r}\right)^{3 / 2}-\left(1+\tan ^{2} \theta_{g}\right)^{3 / 2}} \\
& -\frac{\tan ^{2|n|} \theta_{g}\left(1+|n|^{-1}+\tan ^{2} \theta_{g}\right)}{\left(1+\tan ^{2} \theta_{r}\right)^{3 / 2}-\left(1+\tan ^{2} \theta_{g}\right)^{3 / 2}}
\end{aligned}
$$

where one needs to multiply $\left\langle D_{\alpha \alpha, \pm n}^{-} / p^{2}\right\rangle_{\theta^{r}}^{\theta_{r}}$ by $\pi / 2$ for $|n|=1$.

[18] The full pitch angle diffusion coefficient is obtained after integration over bounce motion [Lyons et al., 1972]:

$$
\left\langle D_{\alpha \alpha}\right\rangle_{B}\left(\alpha_{0}\right)=\int_{\lambda_{\text {MIN }}}^{\lambda_{\text {MAX }}} \frac{D_{\alpha \alpha}(\alpha)}{T\left(\alpha_{0}\right)} \frac{\cos \alpha}{\cos ^{2} \alpha_{0}} \cos ^{7} \lambda d \lambda,
$$

where the bounce period is taken for simplicity as $\left\langle T\left(\alpha_{0}\right)\right\rangle \sim 1$ throughout this paper.

[19] The integrand in equation (6) with equation (3) is proportional to $\Omega_{c}^{3 / 2} \cos ^{7} \lambda \sim \Omega_{c}^{1 / 2}$, meaning only a weak dependence on latitude. Since $\lambda_{\mathrm{MAX}}$ is generally small $\left(<30^{\circ}\right)$ in this range of pitch angles [Mourenas et al., 2012], the bounce-integral can be roughly approximated by the value of its integrant at $\lambda_{R} \sim 0$ multiplied by the domain $\lambda_{\mathrm{MAX}}-\lambda_{\mathrm{MIN}} \sim \lambda_{\mathrm{MAX}}$. With $\Delta \omega / \omega_{m} \sim 0.5$, it leads to a first-order estimate:

$$
\left\langle\frac{D_{\alpha \alpha, \pm n}^{+}}{p^{2}}\right\rangle_{B} \approx \frac{B_{w}^{2}}{B_{0}^{2}} \frac{\Omega_{c 0} \lambda_{\mathrm{MAX}}\left(1+\omega_{m}^{2} / \Omega_{c 0}^{2}\right)}{4 \gamma\left(p \varepsilon_{m 0}\right)^{2} \cos ^{2} \alpha_{0} \sin \alpha_{0}},
$$

with approximately $4 N_{r}$ resonances contributing, where $2 N_{r} \sim 4 p \cos \alpha_{0}\left(\omega_{m} \Omega_{p e} / \Omega_{c 0}^{2}\right)$ is the number of (positive and negative) resonances existing over the whole large- $\theta$ range from $\theta_{g}$ to $\theta_{r}$, obtained from equation (2) taken at $\theta_{g}(\lambda \sim 0)$ [Mourenas et al., 2012]. The frequency-averaged maximum latitude for resonance over the same $\theta$-range can be written as $\lambda_{\mathrm{MAX}} \sim(\sqrt{2} / 6) \min \left(1,1 / \tan \alpha_{0}\right)$ for $\Delta \omega / \omega_{m} \sim 0.5$ [see Mourenas et al., 2012]. For $N_{r}>1$ and in the pitch angle range $\arcsin \left(\omega_{m} / \Omega_{c 0}\right) \sim 20^{\circ}<\alpha_{0}<70^{\circ}$, the total bounceaveraged pitch angle diffusion coefficient $D^{+}$can be estimated by simply multiplying expression (7) by $4 N_{r}$.

[20] At small equatorial pitch angles near the loss cone edge $\left(\alpha_{R}<\pi / 2-\theta_{r}\right)$, the integrand in equation (6) with equation (5) varies roughly like $\Omega_{c}^{3|n|-7 / 9}$. This leads to the following first-order estimate (see Appendix A):

$$
\begin{aligned}
\left\langle\frac{D_{\alpha \alpha, \pm n}^{-}}{p^{2}}\right\rangle_{B} \approx & \frac{B_{w}^{2}}{B_{0}^{2}} \frac{3\left(1+\omega_{m}^{2} / \Omega_{c 0}^{2}\right)}{8 \gamma p^{2} \varepsilon_{m 0}^{2} \cos ^{3} \alpha_{0}} \\
& \times \frac{\Omega_{c 0}^{2|n|} \sin ^{2|n|-2} \alpha_{0}}{\omega_{m}^{2|n|-1}} \int_{\lambda_{m}}^{\lambda_{+}} \frac{\Omega_{c}^{3|n|-7 / 9}(\lambda)}{\Omega_{c 0}^{3|n|-7 / 9}} d \lambda
\end{aligned}
$$

[21] The latitude bounds of integration $\lambda_{+} \sim 40^{\circ}$ and $\lambda_{m}$ are provided in Appendix A, where it is shown that for $N_{r}>2$ and with $\Delta \omega / \omega_{m} \sim 0.5$, equation (8) leads finally to

$$
\left\langle\frac{D_{\alpha \alpha, \pm n}^{-}}{p^{2}}\right\rangle_{B} \approx \frac{B_{w}^{2}}{B_{0}^{2}} \frac{3\left(1+\omega_{m}^{2} / \Omega_{c 0}^{2}\right) \omega_{m}^{13 / 27} \Omega_{c 0}^{14 / 27} \lambda_{M M}}{8 \gamma p^{2} \varepsilon_{m 0}^{2} \cos ^{3} \alpha_{0} \sin ^{41 / 27} \alpha_{0}},
$$

where $\lambda_{M M}$ is given by equation (A3). The diffusion coefficients in equation (9), which were not explicitly provided in Mourenas et al. [2012], are roughly independent of $n$. Once again, the total bounce-averaged pitch angle diffusion coefficient $D^{-}$resulting from the sum over $n$-resonances can be estimated by multiplying expression (9) by $4 N_{r}$. Comparing equation (9) for $D^{-}$with expression (7) for $D^{+}$, it is clear also that $D^{-}$increases faster as $\alpha_{0}$ decreases toward the loss cone angle, roughly like $1 / \sin ^{2} \alpha_{0}$, which explains the steep increase of diffusion rates at very small pitch angle obtained in numerical simulations by Artemyev et al. [2012a] and Mourenas et al. [2012] for constant density.

[22] As concerns momentum diffusion, Lyons [1974] has shown [see also Glauert and Horne, 2005; Albert, 2005] that the local $D_{p p}$ coefficients can be directly calculated from the 
local pitch angle diffusion coefficients $D_{\alpha \alpha}$ in equation (3) as

$$
D_{p p, \pm n}=D_{\alpha \alpha, \pm n} \frac{\sin ^{2} \alpha \cos ^{2} \alpha}{\left(n \Omega_{c} / \gamma \omega_{m}+\sin ^{2} \alpha\right)^{2}},
$$

and the bounce-integrated momentum diffusion coefficients read as

$$
\left\langle D_{p p, \pm n}\right\rangle_{B}\left(\alpha_{0}\right)=\int_{\lambda_{\text {MIN }}}^{\lambda_{\text {MAx }}} D_{p p, \pm n}(\alpha) \frac{\sqrt{1+3 \sin ^{2} \lambda} \cos \lambda d \lambda}{T\left(\alpha_{0}\right) \cos \alpha} .
$$

[23] Assuming $|n| \Omega_{c} / \gamma \omega_{m}>\sin ^{2} \alpha_{0}$, a careful comparison of equation (11) using expression (10) with equation (6) shows that the integrands of equations (11) and (6) vary then very similarly as a function of $\lambda$ for low to moderate resonant latitudes $\lambda<40^{\circ}$ where the chorus waves are present with high amplitudes [Artemyev et al., 2012b]. Since the energy diffusion coefficients read as $D_{E E} / E^{2}=\left(1+\gamma^{-2}\right)$ $D_{p p} / p^{2}$ [see Glauert and Horne, 2005, equation (C5)], the ratio of the bounce-averaged energy and pitch angle coefficients can be rewritten as

$$
\frac{\left\langle D_{E E, \pm n} / E^{2}\right\rangle_{B}\left(\alpha_{0}\right)}{\left\langle D_{\alpha \alpha, \pm n} / p^{2}\right\rangle_{B}\left(\alpha_{0}\right)} \approx \frac{\omega_{m}^{2} \sin ^{2} \alpha_{0} \cos ^{2} \alpha_{0}(\gamma+1)^{2}}{n^{2} \Omega_{c 0}^{2}} .
$$

[24] For $|n| \Omega_{c} / \gamma \omega_{m}>\sin ^{2} \alpha_{0}$, equation (12) is actually equivalent to equation (11) in the work of Albert [2010] obtained in the limit of monochromatic whistler waves (likewise, we assume here narrowband waves with $\left.\Delta \omega / \Omega_{c} \ll 1\right)$.

[25] Considering the $D^{+}$part, the most important resonances (for which resonance occurs over the whole large- $\theta$ range from $\theta_{g}$ to nearly $\theta_{r}$ ) are such that $|n| \geq N_{r} / 2$ with $\Delta \omega / \omega_{m} \sim 0.5$ for typical chorus waves dayside distributions. At smaller $|n|$, indeed, resonance occurs farther and farther away from the equator, in which case the latitude range of resonance decreases [Mourenas et al., 2012]. Since the total $\left\langle D_{\alpha \alpha}\right\rangle_{B}$ is roughly the sum of the $2 N_{r}$ resonances of similar magnitudes, neglecting this decrease is not unreasonable, inducing only small errors. For instance, suppressing totally the resonances $|n| \leq 2$ for $N_{r}=5$ or 10 would lead to a reduction of the total $\left\langle D_{\alpha \alpha}\right\rangle_{B}$ by $20 \%$ or $10 \%$, respectively, which is quite small. On the other hand, the total $\left\langle D_{E E}\right\rangle_{B}$ derived from equation (12) is proportional to the sum of $1 / n^{2}$ terms of contributing resonances, since one has generally $|n| \Omega_{c} / \gamma \omega_{m}>\sin ^{2} \alpha_{0}$ for $|n|>N_{r} / 2$. In the same examples as before, suppressing resonances $|n| \leq 2$ for $N_{r}=5$ or 10 would now diminish the total $\left\langle D_{E E}\right\rangle_{B}$ by $85 \%$ or $80 \%$, respectively, which is clearly a very strong effect. Actually, resonances $|n|<N_{r} / 2$ are not totally suppressed, being reduced by factors $\sim 1 / 3$ to $1 / 10$ typically due to the reduction of the latitude range of resonance over the whole large- $\theta$ range [Mourenas et al., 2012]. Nevertheless, we shall hereafter assume for the sake of simplicity that most resonances $|n|<N_{r} / 3$ are totally suppressed for $N_{r}>3$, which is a reasonable firstorder estimate. Since the sum over $n$ gives a multiplicative factor $\sim 2 \sum n^{-2}=\pi^{2} / 3$ to the individual momentum coefficient $D_{n}$, a first-order estimate of this multiplicative factor for $N_{r}>2$ can be taken as $\sim 2 \pi^{2} /\left(3 N_{r}\right)$, because $\sum_{N_{r} / 3}^{\infty} n^{-2} \approx 3 / N_{r}$. Comparing the total $D^{+}$pitch angle and energy diffusion rates yields simply

$$
\begin{aligned}
\frac{\left\langle D_{E E}^{+} / E^{2}\right\rangle_{B}\left(\alpha_{0}\right)}{\left\langle D_{\alpha \alpha}^{+} / p^{2}\right\rangle_{B}\left(\alpha_{0}\right)} & \approx \frac{\sin ^{2} \alpha_{0} \cos ^{2} \alpha_{0}(\gamma+1)^{2}}{\Omega_{c 0}^{2} / \omega_{m}^{2}} \times \frac{\sum_{N_{r} / 3}^{\infty} n^{-2}\left\langle D_{\alpha \alpha, \pm n}\right\rangle_{B}}{\sum_{1}^{\infty}\left\langle D_{\alpha \alpha, \pm n}\right\rangle_{B}} \\
& =\frac{\sin ^{2} \alpha_{0} \cos ^{2} \alpha_{0}(\gamma+1)^{2}}{\Omega_{c 0}^{2} / \omega_{m}^{2}} \frac{\left(\pi^{2} / 3 N_{r}\right)}{2 N_{r}} \\
& =\frac{\pi^{2} \Omega_{c 0}^{2}(\gamma+1)}{24 \Omega_{p e}^{2}(\gamma-1)} \sin ^{2} \alpha_{0}, \quad N_{r}>2
\end{aligned}
$$

where we use $N_{r}=2 p \cos \alpha_{0}\left(\omega_{m} \Omega_{p e} / \Omega_{c 0}^{2}\right)$. The ratio of energy and pitch angle diffusion rates in equation (13) increases as equatorial pitch angle $\alpha_{0}$ increases for $\sin \alpha_{0}>\omega_{m} / \Omega_{c 0}$, corresponding principally to $D^{+}$[Mourenas et al., 2012]. The ratio (13) also happens to be independent of energy at high energy $E>1 \mathrm{MeV}$, which agrees well with full numerical simulations (see Section 3). For $N_{r}>2$, the corresponding analytical estimate of the bounce-averaged energy diffusion coefficient is obtained from equations (7) and (13) in the range $30^{\circ}<\alpha_{0}<90^{\circ}$ :

$$
\begin{aligned}
\left\langle\frac{D_{E E}^{+}}{E^{2}}\right\rangle_{B} & \approx \frac{\pi^{2}}{24} \frac{\Omega_{c 0}^{2}}{\Omega_{p e}^{2}} \sin ^{2} \alpha_{0} N_{r}\left\langle\frac{D_{\alpha \alpha, \pm 1}}{p^{2}}\right\rangle_{B} \\
& \approx \frac{B_{w}^{2}}{B_{0}^{2}} \frac{\Omega_{c 0}^{4}\left(1+\omega_{m}^{2} / \Omega_{c 0}^{2}\right)(\gamma+1)}{5 \Omega_{p e}^{3} \gamma(\gamma-1) \sqrt{\gamma^{2}-1}} \operatorname{Min}\left[\tan \alpha_{0}, 1\right] .
\end{aligned}
$$

[26] The bounce-averaged energy diffusion rate given by equation (14) increases with $\alpha_{0}$ like $\tan \alpha_{0}$ up to $\alpha_{0} \sim 35^{\circ}$, above which limit it remains constant.

[27] For $\alpha_{0}<35^{\circ}$ however, the $D^{-}$part of the $\theta$-integral must be also included. Now, all the resonances $|n|<N_{r}$ contribute due to the new term $\Omega_{c}^{3|n|-7 / 9}(\lambda) / \Omega_{c 0}^{3|n|}$ in equation (8). The sum over $n$ with equation (12) gives a multiplicative factor $2 \Sigma n^{-2}=\pi^{2} / 3$ to the individual momentum coefficient $D_{n}$. However, only $|n|=1$ to $6-7$ resonances are here contributing significantly to $D_{E E}$, with rapidly decreasing magnitude as $|n|$ grows. Thus, $|n| \sim 3$ can be used as a rough estimate to calculate the lower-bound of latitudinal integration $\lambda_{m}(n=3)$ (see Appendix A). This bound $\lambda_{m}$ was taken as zero in the $D^{+}$part because high- $|n|$ resonances are then more important. It yields

$$
\frac{\left\langle D_{E E}^{-} / E^{2}\right\rangle_{B}\left(\alpha_{0}\right)}{\left\langle D_{\alpha \alpha}^{-} / p^{2}\right\rangle_{B}\left(\alpha_{0}\right)} \approx \frac{\pi^{2}(\gamma+1)^{3 / 2} \omega_{m} \sin ^{2} \alpha_{0}}{24 \Omega_{p e} \cos \alpha_{0} \sqrt{\gamma-1}} \frac{\lambda_{M M}-\left.\lambda_{m}\right|_{n=3}}{\lambda_{M M}} .
$$

[28] The ratio of energy and pitch angle diffusion rates in equation (15) always increases as equatorial pitch angle $\alpha_{0}$ increases in the corresponding range $\sin \alpha_{0}<2 \omega_{m} / \Omega_{c 0}$. It depends strongly on energy. The corresponding analytical estimate of the bounce-averaged energy diffusion coefficient is obtained from equations (9) and (15) in the range $0^{\circ}<\alpha_{0}<45^{\circ}$ for $N_{r}>2$ :

$$
\begin{aligned}
\left\langle\frac{D_{E E}^{-}}{E^{2}}\right\rangle_{B} \approx & \frac{B_{w}^{2}}{B_{0}^{2}} \frac{\Omega_{c 0}^{41 / 27} \omega_{m}^{40 / 27} \sin ^{13 / 27} \alpha_{0}(\gamma+1)}{\left(8 / \pi^{2}\right) \Omega_{p e}^{2} \gamma(\gamma-1)} \\
& \times\left(\lambda_{M M}-\left.\lambda_{m}\right|_{n=3}\right)\left(1+\omega_{m}^{2} / \Omega_{c 0}^{2}\right)
\end{aligned}
$$


[29] Nevertheless, the complex integrations over $\theta$ and $\lambda$ performed in Appendix A to derive equation (16) are probably too roughly approximated at very small $\alpha_{0}$ where resonance occurs at very high latitudes near the resonance cone. Moreover, the simplified dispersion relation used here becomes much less reliable at such high latitudes $\lambda>30^{\circ}$ Finally, equations (14)-(16) should not be used at low energy beyond their validity limit given by $N_{r}>2$.

[30] The above estimates have been derived for highintensity chorus waves present up to latitudes of about $40^{\circ}$, corresponding to an important contribution of very oblique waves. Such a wave distribution is actually representative of the dawn/day side chorus distribution measured by CLUSTER (see detailed distributions in Agapitov et al. (unpublished manuscript, 2012) and Artemyev et al. [2012b]). On the dusk/night side, however, high-intensity chorus waves are mainly present at latitudes lower than $20^{\circ}$, [Artemyev et al., 2012b]. The impact of very oblique waves is then expected to be much smaller. Therefore, it is interesting to estimate also the energy diffusion rate in the case of a Gaussian wave-normal distribution $g(\theta)=\exp \left(-\tan ^{2} \theta /\right.$ $\left.\tan ^{2} \Delta \theta\right)$ such that $\Delta \theta<45^{\circ}$. In this case, Mourenas and Ripoll [2012, equation (30)] giving an analytical estimate of the bounce-averaged pitch angle diffusion coefficient at large pitch angles $\alpha_{0}>30^{\circ}$, is still approximately valid for lower-band chorus [Mourenas et al., 2012]. About $n \Delta \omega /$ $\omega_{m} \sim\left(p \varepsilon_{m 0} \cos \alpha_{0}\right) \Delta \omega / \omega_{m}$ cyclotron resonances are then contributing to the scattering rate at a given $\alpha_{0}$. Summation over $n$ of equation (13) yields a multiplicative factor $\sum n^{-2} \sim \Delta \omega / n \omega_{m}$. For $\cos \alpha_{0}>\left(1-\gamma \omega_{m} / \Omega_{c 0}\right)\left(1-\omega_{m} /\right.$ $\left.\Omega_{c 0}\right)^{1 / 2} /\left(p \varepsilon_{m 0}\right)$ and over most of the large pitch angle range, the bounce-averaged energy diffusion rate can then be written as:

$$
\left\langle\frac{D_{E E}^{+ \text {small }-\theta}}{E^{2}}\right\rangle_{B} \approx \frac{B_{w}^{2}}{B_{0}^{2}} \frac{\Omega_{c 0}^{4} \sin \alpha_{0} \sqrt{(\gamma+1) \omega_{m} / \Omega_{c 0}}}{2 \tan \Delta \theta \Omega_{p e}^{3} T\left(\alpha_{0}\right) \gamma(\gamma-1)^{3 / 2}} .
$$

[31] From equations (14) and (17) for $\tan \Delta \theta \sim 0.6$, it is easy to see that an oblique (dayside-like) chorus distribution leads to energy diffusion rates reduced by an almost constant factor of about $2.5 / \sin \alpha_{0}$ as compared to nearly-parallel (nightside-like) waves at pitch angles $\alpha_{0}>45^{\circ}$. However, these estimates have been derived for nearly uniform latitudinal distributions of wave intensities, while Shprits et al. [2006] have shown that this latitudinal distribution may also be an important parameter in determining scattering rates. Therefore, a comparison of our simplified analytical estimates with energy diffusion rates evaluated numerically with actual, measured wave intensity distributions varying with latitude [Artemyev et al., 2012b] will be provided in Section 3.

[32] From equations (14), (16), and (17) electron energy diffusion $D_{E E} / E^{2}$ varies like

$$
\begin{aligned}
& \left\langle\frac{D_{E E}}{E^{2}}\right\rangle_{B} \sim \frac{\Omega_{c 0}^{2} \sqrt{\gamma+1}}{\Omega_{p e}^{3} \gamma(\gamma-1)^{3 / 2}}, \quad N_{r}>2 \quad \alpha_{0}>45^{\circ}, \\
& \left\langle\frac{D_{E E}}{E^{2}}\right\rangle_{B} \sim \frac{\omega_{m}^{3 / 2}(\gamma+1)}{\Omega_{p e}^{2} \Omega_{c 0}^{1 / 2} \gamma(\gamma-1)}, \quad N_{r}>2 \quad \alpha_{0}<10^{\circ} .
\end{aligned}
$$

[33] For $\Omega_{p e} / \Omega_{c 0} \geq 4$ at low $L>3.5$ in typical density profiles in the outer belt [Sheeley et al., 2001] and at energies $E>0.5 \mathrm{MeV}$, the ratio $\left\langle D_{E E} / E^{2}\right\rangle_{B} /\left\langle D_{\alpha \alpha} / p^{2}\right\rangle_{B}$ is independent of energy and $\left\langle D_{E E} / E^{2}\right\rangle_{B} \propto 1 / E^{2}$ at large equatorial pitch angles $\alpha_{0}>40^{\circ}$. For a dipolar geomagnetic field, an average plasma density given by the model of Sheeley et al. [2001], and for fixed wave intensities (not varying with $L$ ), the acceleration rate at large equatorial pitch angles is then nearly independent of $L$ at high enough energy such that $N_{r}>2$. Peaks of acceleration should then occur mainly on the night-side in regions of lower-than-average plasma density (at $L \sim 5$ just outside the plasmasphere [Horne et al., 2005]) and/or in regions of enhanced wave power. At smaller equatorial pitch angles $\alpha_{0}<20^{\circ}$ and for fixed ratio $\omega_{m} / \Omega_{c 0}$, the ratio $\left\langle D_{E E} / E^{2}\right\rangle /\left\langle D_{\alpha \alpha} / p^{2}\right\rangle$ is proportional to $E$ at high energy and $\left\langle D_{E E} / E^{2}\right\rangle_{B} \propto L / E$. Consequently, energy diffusion is then stronger at lower density, energy, but higher $L$-shells for an average density model [Sheeley et al., 2001] and fixed wave intensities (not varying with $L$ ). In reality, energy diffusion should again be stronger in regions of lower-than-average plasma density and/or enhanced chorus wave power. Stronger energy diffusion at lower densities was already pointed out by Horne and Thorne [2003] on the basis of numerical simulations for parallel waves. The approximate analytical model (14)-(16)(17) provides explicitly the dependence of the energy diffusion rate on each parameter, allowing to perform quick estimations of the sensitivity of electron energization to the many different plasma, particle, and wave parameters, which exhibit considerable variability as a function of geomagnetic activity [Horne and Thorne, 2003; Li et al., 2007; Miyoshi and Kataoka, 2011; Kellerman and Shprits, 2012].

\section{Comparison of Analytical Estimates With Full Numerical Simulations and Discussion}

[34] The analytical estimates (14)-(17) of electron energy diffusion rates are compared with full numerical calculations in the case of lower-band chorus whistler waves dominant on the day-side in the outer radiation belt, where $\omega_{m} / \Omega_{c 0} \sim 0.35$ on average [Agapitov et al., 2011]. We use the numerical scheme of calculation of diffusion rates described by Glauert and Horne [2005]. However, we also take into account the effect of oblique wave propagation by incorporating experimental statistics [Agapitov et al., 2011] into the numerical scheme (see details in Artemyev et al. [2012a, 2012b]). A realistic Gaussian wave-normal angle distribution is assumed in numerical calculations: $g(\theta)=$ $\exp \left(-\left(\tan \theta-X_{m}\right)^{2} / X_{w}^{2}\right)$. The position of its peak $X_{m}=X_{m}(\lambda)$ as well as its variance $X_{w}=X_{w}(\lambda)$ are written as polynomial functions of latitude obtained by fitting chorus statistics from CLUSTER [Artemyev et al., 2012a; Mourenas et al., 2012]. Storm-time chorus waves in the dayside plasma trough are assumed with average amplitudes of $100 \mathrm{pT}$ as in Shprits et al. [2007] on the basis of CRRES observations. We take also $L \sim 4$ and a ratio $\Omega_{p e} / \Omega_{c 0} \sim 4.5$ corresponding to average conditions in the outer belt, where this ratio varies between about 2 and 10 [Horne et al., 2005].

[35] Let us start with the case of significantly oblique chorus waves, which corresponds mainly to the dawn/day sector. There is an excellent agreement between the analytical estimates (13) and (15) of the ratio of the $\left\langle D_{E E}\right\rangle_{B}$ and $\left\langle D_{\alpha \alpha}\right\rangle_{B}$ coefficients and the full numerical solutions in Figure 1 , for $E=0.1 \mathrm{MeV}$ to $5 \mathrm{MeV}$, which requires up 

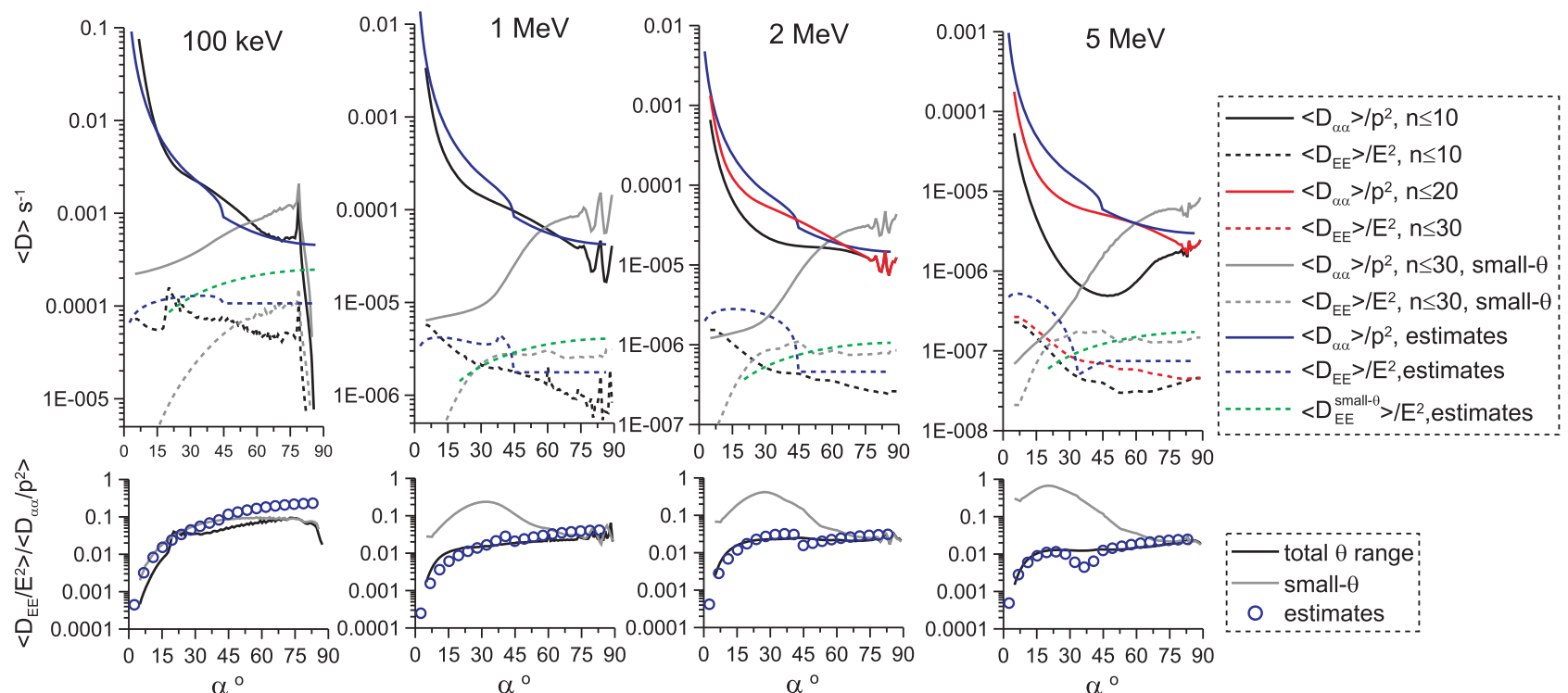

Figure 1. (top) Numerical pitch angle (solid lines) and energy (dashed lines) diffusion rates for $100 \mathrm{pT}$ storm-time lower-band oblique (black, $X_{m, w}=X_{m, w}(\lambda)$ ) or nearly parallel (grey, $X_{m}=0$ and $X_{w}=0.577$ ) chorus waves at $\Omega_{p e} / \Omega_{c 0}=4.5$ and $L \sim 4$. Dotted and solid blue curves show respectively the analytical estimates of the energy and pitch angle diffusion rates for oblique (dayside) waves, while the green dotted curves show the analytical energy diffusion rates for moderately oblique (nightside) waves. (bottom) The ratios of energy to pitch angle diffusion rates.

to 20 positive and negative harmonics evaluations. It shows that essential features of energy and pitch angle diffusions are well taken into account. In Figure 1, the discrepancy between analytical and numerical pitch angle diffusion rates is always smaller than a factor of 2 for $E=0.1 \mathrm{MeV}$ to $5 \mathrm{MeV}$, which is actually more than thrice smaller than the sum of the uncertainties due to density models, wave intensity variability, and latitudinal distribution of waves [see Sheeley et al., 2001; Artemyev et al., 2012b; Agapitov et al., unpublished manuscript, 2012]. The discrepancy between analytical and numerical energy diffusion rates remains also smaller than a factor 2.5 in general. At low energy $E=$ $0.1 \mathrm{MeV}$, however, two peaks can be noticed in the numerical energy diffusion rate near $20^{\circ}$ and $75^{\circ}$, coming from the Landau resonance. Landau resonance was neglected in the present analytical estimates, while it becomes important in the full numerical results at such low energy. Moreover, equations (14) and (16) are used beyond their validity limit given by equation (A6) at low energy $E=0.1 \mathrm{MeV}$ in this case, since $N_{r}$ becomes then smaller than 2. For $N_{r}<2$, the sum over $n$ of the individual energy diffusion coefficients is actually overestimated in equations (13) to (16). A more accurate estimate for $D_{E E}^{-}$in the range $1 /\left(1+\Delta \omega / \omega_{m}\right)<N_{r}<2$ would be $2 \sum n^{-2} \sim\left(\pi^{2} / 3\right) \min \left(1, N_{r} / 2\right)$. This would lead to a multiplicative factor $\sim 0.5$ at $E=0.1 \mathrm{MeV}$ in Figure 1, putting the analytical energy diffusion rate in even better agreement with the numerical solution for cyclotron resonances $(|n| \geq 1)$. Note also that the ratio $\Omega_{c}^{2}\left(\lambda_{R}\right) / \Omega_{c 0}^{2} \sim$ $N_{r}\left(\omega_{m}\right) \propto \sqrt{\gamma^{2}-1}$ for $\theta_{R} \sim \theta_{g}$ decreases at lower energy. High latitudes then correspond to resonant $\theta_{R}$ values closer to $\theta_{r}$ than at larger energy. This is further confirmed in Appendix B, where it is shown that resonance for $\omega / \Omega_{c 0}>0.2$ occurs at lower energy when $\theta_{R}$ tends toward $\theta_{r}$. The simplified dispersion used and/or the rough approximation of the actual integral over $\theta$ and latitude made in Appendix A could become too crude very near $\theta_{r}$. At high energy $E>2 \mathrm{MeV}$, conversely, the second validity limit given by equation (A6) is crossed at moderate values of $\alpha_{0}$, meaning that our analytical diffusion coefficient estimates are then valid only at very small equatorial pitch angles $\alpha_{0}<5^{\circ}$ (for $D^{-}$) or $\alpha_{0}>40^{\circ}$ (for $D^{+}$). This may explain the larger discrepancy observed at intermediate pitch angles $\alpha_{0}=20^{\circ}$ to $40^{\circ}$ between analytical estimates and full numerical solutions at $2 \mathrm{MeV}$ in Figure 1. Finally, considering now only small- $\theta$ waves corresponding to nightside chorus distributions, the comparison between estimate (17) of $\left\langle D_{E E}\right\rangle_{B}$ and the full numerical solution in Figure 1 shows also a good agreement over the range of validity of the analytical estimate $\left(30^{\circ}<\alpha_{0}<\arccos \left(1 / p \varepsilon_{m 0}\right)\right.$ and $\left.p \varepsilon_{m 0}>1\right)$.

[36] It is worth noting that the approximation of a constant wave amplitude $B_{w}$ used in the analytical expressions of diffusion rates as well as in the numerical simulations in Figure 1 should be considered only as a first order approximation. Spacecraft observations actually show a significant variation of $B_{w}$ with latitude $\lambda$ [see, e.g., Meredith et al., 2001; Horne et al., 2005; Artemyev et al., 2012b; Agapitov et al., unpublished manuscript, 2012]. Moreover, for a given magnetic latitude, the distribution of wave amplitudes can be substantially different from a Gaussian distribution, with corresponding power law tails of probability of large $B_{w}$ [Cully et al., 2008; Agapitov et al., 2011; Bunch et al., 2012]. Therefore, it is more reasonable to consider the dependence of $B_{w}$ on $\lambda$ for various levels of probability, as it was done for pitch angle diffusion coefficients by Artemyev et al. [2012b]. Here we use CLUSTER observations to define functions $B_{w}(\lambda)$ for two MLT sectors: the dawn/day sector corresponds to MLT from 02.00 to 14.00 , while the dusk/night sector corresponds to MLT from 14.00 to 02.00 . 

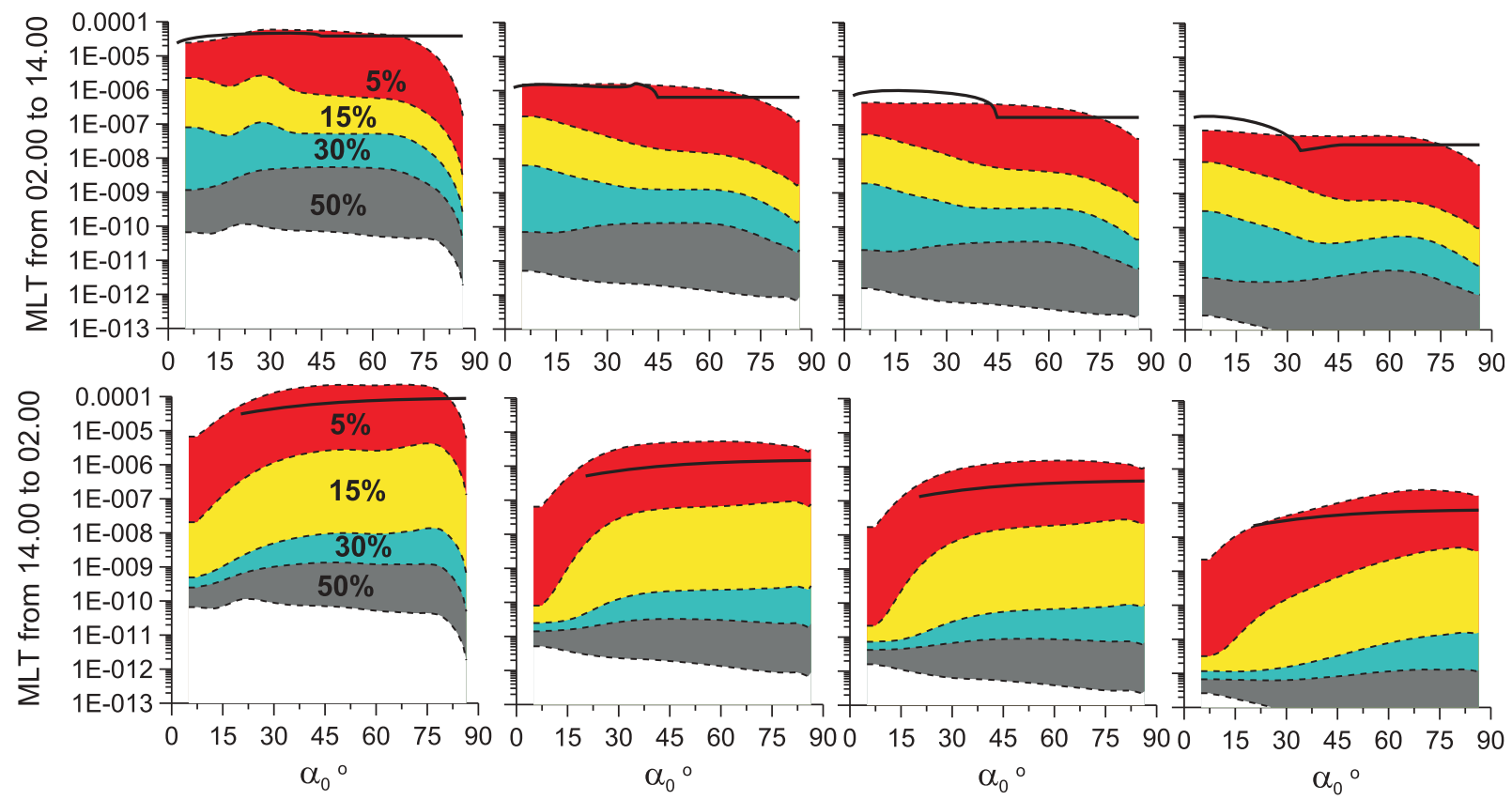

Figure 2. Distribution of energy diffusion coefficients $\left\langle D_{E E} / E^{2}\right\rangle \mathrm{s}^{-1}$ for $\Omega_{p e} / \Omega_{c 0}=4.5$ and four energies ( $E=0.1,1,2$, and $5 \mathrm{MeV}$ from left to right). Red color corresponds to the range of $\left\langle D_{E E} / E^{2}\right\rangle$ variation for the most intense waves with $5 \%$ probability; $\left\langle D_{E E} / E^{2}\right\rangle$ can be found inside yellow, blue and grey ranges for less intense waves ranges corresponding to $15 \%, 30 \%$ and $50 \%$ probabilities, respectively. For the calculation of $\left\langle D_{E E} / E^{2}\right\rangle$ the effect of $B_{w}$ variation with $\lambda$ is also taken into account. The $B_{w}$ distributions correspond to high geomagnetic activity $K_{p}>5$ (see details of chorus distributions in Artemyev et al. [2012b]). The (top) dawn/day and (bottom) dusk/night sectors. Black solid curves show $\left\langle D_{E E} / E^{2}\right\rangle$ estimates (14), (16), and (17) for $60 \mathrm{pT}$ chorus waves.

For each sector we fit dependencies $B_{w}(\lambda)$ by polynomials and calculate diffusion rates $\left\langle D_{E E} / E^{2}\right\rangle$ (details of this fitting and corresponding functions $B_{w}(\lambda)$ for various probability levels can be found in Artemyev et al. [2012b]). Figure 2 shows distributions of $\left\langle D_{E E} / E^{2}\right\rangle$ for $\Omega_{p e} / \Omega_{c 0}=4.5$ at four energies. Each color is used for the related probability to observe $\left\langle D_{E E} / E^{2}\right\rangle$ inside the corresponding range. The comparison of Figures 1 and 2 demonstrates the effect of $B_{w}$ variation with $\lambda$, since the range of $\left\langle D_{E E} / E^{2}\right\rangle$ variations in Figure 2 corresponds to a realistic distribution of wave amplitudes $B_{w}$.

[37] In the dawn/day sector where the most intense waves are measured between latitudes $15^{\circ}$ and $40^{\circ}$, it is worth noting that the energy diffusion rate $\left\langle D_{E E} / E^{2}\right\rangle$ calculated with $100 \%$ of the distribution of wave intensity, i.e. with the $5 \%$ most intense waves fully included, is comparable with $\left\langle D_{E E} / E^{2}\right\rangle$ calculated under the assumption that $B_{w}=100 \mathrm{pT}$. It means that only about $5 \%$ of the waves have amplitudes of $100 \mathrm{pT}$ or more, but also that the corresponding integrated (over intensity) part of the wave intensity distribution corresponds more or less to waves of 60 to $100 \mathrm{pT}$ effective average amplitude. Taking into account only $95 \%$ of the less intense observed waves, the corresponding energy diffusion rates are already one order of magnitude smaller than $\left\langle D_{E E} / E^{2}\right\rangle$ for $B_{w}=100 \mathrm{pT}$. Similar results were obtained for pitch angle diffusion rates [Artemyev et al., 2012b]. It means that the high-intensity range in the wave distribution actually controls the rate of diffusion, because the decrease of the probability of occurrence with intensity is not quick enough to allow neglecting this high-intensity part. Moreover, we would like to point out that the weight of high-intensity waves in energy diffusion rates in Figure 2 is probably amplified by the fact that high-intensity waves have generally been measured on CLUSTER at latitudes where oblique wave diffusion efficiency is also the highest regardless of wave intensity, i.e. between $\lambda_{M}(n=1) \sim 2^{1 / 2} \sqrt{\left(N_{r}+N_{r} \Delta \omega / \omega_{m}\right)^{1 / 18}-1}>0$ and $\lambda_{M M}<35^{\circ}$ (see Appendix A and also the $\lambda<30^{\circ}$ curves in Mourenas et al. [2012, Figure 8]). On the dayside, the analytical estimates (14) and (16) of the energy diffusion rates derived for mainly oblique waves are in good agreement with the numerical rates calculated with realistic chorus distributions for $E>100 \mathrm{keV}$.

[38] In the dusk/night sector where the most intense waves are measured between latitudes $0^{\circ}$ and $20^{\circ}$, the energy diffusion rate $\left\langle D_{E E} / E^{2}\right\rangle$ calculated with $100 \%$ of the distribution of wave intensity is comparable with $\left\langle D_{E E} / E^{2}\right\rangle$ calculated under the assumption that $B_{w}=60 \mathrm{pT}$ with a nearly parallel wave-normal distribution (see Figure 2). In fact, the most intense recorded waves being confined to lower latitudes, they are less oblique than in the dawn/day sector. The corresponding analytical estimate (17) of the energy diffusion rate derived for moderately oblique waves is in good agreement with the numerical rate calculated with a realistic chorus distribution, especially at higher energy $E>100 \mathrm{keV}$ and at larger equatorial pitch angles $\alpha_{0}>30^{\circ}$.

[39] The stochastic diffusion of particles by an ensemble of uncorrelated waves usually occurs preferentially toward regions of smaller phase space density [Walt, 1994; Summers et al., 1998; van Milligen et al., 2005]. Based on 
the work of Summers et al. [1998], quasi-linear diffusion of electrons by parallel chorus waves should also occur along so-called resonant diffusion surfaces at a given latitude. Although the study of Summers et al. [1998] was restricted to parallel waves, each $n$-resonance local diffusion characteristic remains qualitatively similar for oblique waves to the first-order one for parallel waves (see Appendix B). The increase of $\theta$ essentially does not change the form of the local resonant curves in the $\left(v_{\perp}, v_{\|}\right)$plane, but for larger $\theta$ values closer to $\theta_{r}$, resonances with higher cyclotron harmonics become available for particles with smaller energy. Locally, quasi-linear energy diffusion could then lead either to acceleration or deceleration of particles, depending on the main direction of pitch angle diffusion (toward larger or smaller pitch angles, respectively) [Summers et al., 1998; Horne and Thorne, 2003], which should be determined by phase space density gradients. However, bounce-averaging over latitudes probably modifies the local diffusion characteristics. Furthermore, actual phase space density gradients result from a complex interplay between quasi-linear pitch angle and energy diffusion, mixed diffusion, radial diffusion, adiabatic transport, and convection, requiring computationally expensive full-scale Fokker-Planck numerical simulations. Nevertheless, looking separately at small and large equatorial pitch angle electrons, our analytical and numerical results can help us to roughly outline some expected features.

[40] First, at large equatorial pitch angles $\alpha_{0}>60^{\circ}$, adiabatic invariance in a dipolar geomagnetic field implies that resonance is confined to a narrow range of latitudes $\left(\lambda<15^{\circ}\right)$ close to the equator [see Mourenas et al., 2012, equation (11)]. There, chorus waves measured by CLUSTER and THEMIS are only moderately oblique on the nightside as well as on the dayside [Agapitov et al., 2011; Li et al., 2011; Artemyev et al., 2012b]. Therefore, the results of previous theoretical developments [Summers et al., 1998; Horne and Thorne, 2003] and Fokker-Planck numerical simulations [Li et al., 2007; Varotsou et al., 2008; Shprits et al., 2009] concerning nearly parallel chorus waves should still roughly apply in this case. It implies that high pitch angle electrons of energy $E>0.1 \mathrm{MeV}$ should be mainly accelerated by the waves, on both the nightside and the dayside. Furthermore, Figures 1 and 2 show that acceleration rates should only be reduced by a factor of 2 on the dayside when using the realistic chorus distributions obtained by CLUSTER instead of parallel waves, while they would remain almost unchanged on the nightside where most intense waves are very moderately oblique. Nightside or dayside acceleration may therefore dominate, depending on the plasma/wave parameters over the respective sectors. However, loss rates are strongly increased on the dayside by oblique waves. This could potentially impact the net acceleration of injected electrons from the magnetotail over the long term.

[41] Let us now consider the opposite case of electrons of small equatorial pitch angles $\alpha_{0}<30^{\circ}$ and let us focus only on the dawn/day sector (numerical calculations show indeed that energy diffusion is very weak at small pitch angles in the dusk/night sector, consistent with estimates for parallel waves). On the dayside, quasi-linear pitch angle scattering is very likely the fastest diffusive/transport process, with timescales ranging from minutes to hours for chorus amplitude $B_{w} \sim 100 \mathrm{pT}$ during periods of important geomagnetic activity and for energies $E=0.05$ to $2 \mathrm{MeV}$ (see Figures 1 and 2) [see also Mourenas et al., 2012, Figure 7; Artemyev et al., 2012b, Figure 3]. Considering an initial dayside electron distribution with loss cone anisotropy, small pitch angle electrons would then mainly diffuse toward the loss cone, before being lost in the ionosphere. Provided that the main qualitative feature of the local resonant diffusion characteristics (i.e. particles loosing energy when diffusing toward the loss cone) is not fundamentally modified by bounce-averaging in the presence of different $n$ resonances, this could correspond to small pitch angle electron deceleration and associated quasi-linear wave growth on the dayside. Consequently, the mechanism of energy transfer from 30-100 keV electrons to higher-energy ones via chorus wave excitation discussed by Horne and Thorne [2003] might be enhanced with realistic dayside chorus distributions, due to the order-of-magnitude increase in pitch angle and energy diffusion rates obtained at moderate pitch angles when including very oblique waves observed at latitudes above $15^{\circ}$ (see Figures 1 and 2). However, full-scale Fokker-Planck numerical simulations of the outer belt will obviously be needed in the future to confirm (or not) the preceding conjectures.

[42] As a matter of fact, the statistical maximum in dayside chorus intensities observed by CLUSTER for $K_{p}>3$ at latitudes between $15^{\circ}$ and $35^{\circ}$ [Artemyev et al., 2012b; Agapitov et al., unpublished manuscript, 2012] can probably be explained by the combined effects of dispersion, refraction, linear growth, Landau damping, and quasi-linear diffusion of low-energy electrons. As a first step, chorus waves can be linearly generated near the equator at a range of wave-normal angles between $0^{\circ}$ and $\theta_{g} \leq 45^{\circ}$, consistent with observed wave-normal distributions at the equator [Trakhtengerts, 1999; Agapitov et al., 2011, also unpublished manuscript, 2012]. Chorus waves should then be refracted by density and magnetic field gradients as they propagate to higher latitudes [Horne and Thorne, 2003; Chum and Santolik, 2005], leading to a progressive increase of their wave-normal angle, generally going above the Gendrinangle at $\lambda>15^{\circ}$ as observed in both ray-tracing simulations [Breuillard et al., 2012] and CLUSTER statistics [Agapitov et al., 2011]. At a given latitude, the actual shape of the wave-normal distribution will be further modified by the competing effects of amplification and damping. Based on numerical calculations in Figures 1 and 2 and on the above analytical estimates, abundant low-energy $(<100 \mathrm{keV})$ electrons with small pitch angles $\alpha_{0}<20^{\circ}$ might transfer their energy to the waves much more efficiently for $\theta>\theta_{g}$ than for smaller wave-normal angles. Consequently, chorus growth due to resonant quasilinear diffusion could be much stronger at latitudes above $10^{\circ}$ where waves are predominantly oblique than near the equator where almost parallel waves dominate. Furthermore, small pitch angle electron diffusion by oblique waves is also stronger (for constant intensity and $\theta$-distribution) between $\lambda_{M}(n=1) \sim 9^{\circ}-18^{\circ}$ and $\lambda_{M M} \sim 35^{\circ}$ for the same typical parameters as in Figures 1 and 2 and $E \leq 0.1 \mathrm{MeV}$. Moreover, waves should propagate (presumably from the equator) while growing or getting damped in the linear and quasi-linear stages. On the nightside, the presence of a strong Landau damping probably accounts for the equatorial confinement of intense waves [ $\mathrm{Li}$ et al., 2011]. 
However, Landau damping is believed to be less important on the dayside [Li et al., 2011]. All these effects concur to produce a sharp increase of wave intensities between $0^{\circ}$ and $15^{\circ}$ of latitude on the dayside. CLUSTER statistics actually show such a steep increase of wave intensities by one to two orders of magnitude between the equator and $\lambda \sim 15^{\circ}$ for the $15 \%$ to $25 \%$ portion of the waves with highest amplitudes [Artemyev et al., 2012b; Agapitov et al., unpublished manuscript, 2012]. The subsequent flattening out and then decrease of intensities at latitudes above $35^{\circ}$ could be partly related to the eventual departure of the waves from their initial field-line or to their reflection when their frequency gets closer to the lower-hybrid frequency. The reduction of diffusion rates (for constant intensity and $\theta$-distribution) above $\lambda_{M M} \sim 35^{\circ}$ may also contribute. Due to their significant perpendicular group velocity $\left(v_{g \perp} \sim\right.$ $\omega / k \sin \theta)$, most of the waves with large wave-normal angles are expected to depart from their original geomagnetic field line after their first travel from low to high latitudes or, if reflected (or else if coming from other $L$-shells), before traveling back to $\lambda<10^{\circ}$. Ray tracing simulations coupled to Fokker-Planck numerical calculations would be required to better assess all these points.

\section{Conclusions}

[43] In this paper, the effects of very oblique chorus waves $\left(\theta>45^{\circ}\right)$ on electron energization in the outer radiation belt have been investigated by means of analytical as well as full numerical calculations in the frame of quasi-linear theory. Recent CLUSTER statistics are used for characterizing the angular distribution of chorus waves as a function of latitude. The analytical estimates of the electron diffusion rates have been derived making use of the Lyons et al. [1972] model with the weighted-average reformulation of Albert [2007], on the approximation of the Bessel functions by their upper envelop [Mourenas and Ripoll, 2012], and on the assumption of a resonance maximum occurring near the mean frequency $\omega_{m}$ (for $\Delta \omega<\omega_{m} / 2$ ). We consider only lower-band $\left(\Omega_{L H} \ll \omega<\Omega_{c} / 2\right)$ oblique chorus whistler waves observed by CLUSTER [Agapitov et al., 2011] in the limit $\Omega_{p e} / \Omega_{c 0} \geq 4$ appropriate for our simplified dispersion relation. A rough agreement is obtained between the analytical model and the full numerical results at energies $E \sim$ $0.1 \mathrm{MeV}$ to $5 \mathrm{MeV}$. At lower energy $E<0.1 \mathrm{MeV}$, Landau resonance becomes more important and the analytical cyclotron energy diffusion rates presented here cannot be used alone anymore. The analytical estimates provide new scaling laws of energization rates as a function of electron energy, plasma density and geomagnetic field amplitude. For very oblique chorus waves corresponding to measured distributions on the dayside, energy diffusion should remain stronger at low energy and at $L$-shells with lower-thanaverage density (just outside the plasmasphere) or with higher-than-average wave intensities. Dayside oblique chorus waves are found to strongly increase the energy diffusion rates of small pitch angle electrons, often by more than one order of magnitude. At large pitch angles, oblique waves lead to reduced energy diffusion as compared to parallel waves. Since the energy lost to the waves by electrons with small equatorial pitch angles should then be augmented, the net effect could be an increase of wave growth provided by intense low energy electron fluxes at $10^{\circ}<\lambda<35^{\circ}$ in the dawn/day sector. Additionally, a subsequent increase of the acceleration of less numerous higher energy electrons slightly closer to the equator could occur. Near the equator, chorus waves are indeed less oblique and more efficient in energizing large pitch angle electrons, especially during periods of magnetic storms and substorms and for reduced plasma density. The maximum of wave intensities between $15^{\circ}$ and $35^{\circ}$ of latitude observed by CLUSTER on the dayside might be closely related to this higher efficiency of diffusion of small pitch angle electrons by oblique waves in this range of latitudes, as well as to a weaker Landau damping than on the nightside. On the nightside, the most intense measured chorus waves are much less oblique and confined to lower latitudes. Analytical estimates of the energy diffusion rates are also provided in this case, showing a good agreement with numerical simulations. The nightside acceleration rates are only slightly modified when using realistic chorus distributions as compared to parallel waves only. Finally, it has also been shown that the high-intensity tail of the non-Gaussian realistic wave distribution measured by CLUSTER actually controls the rate of energy diffusion over the long term.

\section{Appendix A: Bounce-Averaged $D^{-}$Pitch Angle Diffusion Coefficient}

[44] Equation (5) can be easily derived by integration over $\theta$ of Mourenas et al. [2012, equation (C1)] or Albert [2007, equation (7)]. It amounts to integrating $J_{|n|-1}(x) \tan \theta \sqrt{1+\tan ^{2} \theta} d \tan \theta$ with $x \sim n \tan \theta \tan \alpha$ over the full large- $\theta$ domain from $\theta_{g}$ to $\theta_{r}$, which has been shown to contribute the most to diffusion rates [Mourenas et al., 2012]. It is further assumed in equation (5) that resonance exists over this full large- $\theta$ domain and that small-argument classic expansions of the Bessel functions can be used to approximate their variation with $\theta$. Near the loss cone edge (for $\alpha_{0}<\pi / 2-\theta_{g}$ ), first estimates of the latitude bounds of integration in equation (6) with equation (5) can be derived from equation (2) taken at $\theta_{g}\left(\lambda_{R} \sim 0\right)$, yielding $\Omega_{c}^{2}\left(\lambda_{M}\right) /$ $\Omega_{c 0}^{2} \sim\left(1+\Delta \omega / \omega_{m}\right) N_{r}\left(\omega_{m}\right) / n$ and $\Omega_{c}^{2}\left(\lambda_{m}\right) / \Omega_{c 0}^{2} \approx \max$ $\left(1,\left(1-\Delta \omega / \omega_{m}\right) N_{r}\left(\omega_{m}\right) / n\right)$ for $|n| \leq N_{r}\left(\omega_{m}+\Delta \omega\right)$ and $\cos \alpha_{R} \approx \cos \alpha_{0} \approx 1$, where we recall that $N_{r}$ is evaluated at equator with $\theta_{R}=\theta_{g}$ (see details in Mourenas et al. [2012]). It is worth noting that $N_{r}$ is calculated with $\theta_{R}=\theta_{g}$. This gives the range of latitudes over which resonance exists over the full large- $\theta$ domain from $\theta_{g}$ to $\theta_{r}$, as actually assumed to obtain equation (5). At higher latitudes $\lambda>\lambda_{M}$, resonance occurs only closer to $\theta_{r}$ so that $\theta_{g}$ in equation (5) should be replaced by $\theta_{R}(\lambda)>\theta_{g}(\lambda)$ to be more exact. Nevertheless, for $\Delta \omega / \omega_{m} \sim 0.5$ and $N_{r}\left(\omega_{m}\right)>2$, it is not unreasonable to assume that the inequality $\left(\tan \theta_{r}\right)^{2|n|+2}>2\left(\tan \theta_{R}\right)^{2|n|+2}$ stillholds for most latitudes, nearly up to the maximum latitude $\lambda_{+}$where resonance can be reached (for $\theta \sim \theta_{r}$ ). The latter corresponds to the highest latitude where large-amplitude chorus waves are present and it is fixed here at $\lambda_{+} \sim 40^{\circ}$ in accordance with observations from CLUSTER and POLAR spacecraft [Agapitov et al., 2011; Bunch et al., 2012; Agapitov et al., unpublished manuscript, 2012]. 
[45] Then, the complicated ratio of tangents in equation (5), which comes partly from a small-argument expansion of Bessel function $J_{|n|-1}^{2}$, can be very simply approximated by $\sim 1.2\left(\tan \theta_{r}\right)^{2|n|-1}$. It yields a first-order estimate of the bounce-averaged diffusion coefficient near the loss cone edge:

$$
\begin{aligned}
\left\langle\frac{D_{\alpha \alpha, \pm n}^{-}}{p^{2}}\right\rangle_{B} \approx & \frac{B_{w}^{2}}{B_{0}^{2}} \frac{3\left(1+\omega_{m}^{2} / \Omega_{c 0}^{2}\right)}{8 \gamma p^{2} \varepsilon_{m 0}^{2} \cos ^{3} \alpha_{0}} \\
& \times \frac{\Omega_{c 0}^{2|n|} \sin ^{2|n|-2} \alpha_{0}}{\omega_{m}^{2|n|-1}} \int_{\lambda_{m}}^{\lambda_{+}} \frac{\Omega_{c}^{3|n|-7 / 9}(\lambda)}{\Omega_{c 0}^{3|n|-7 / 9}} d \lambda .
\end{aligned}
$$

[46] The actual $\lambda$-integration is not easy to perform, however, because different regimes are successively encountered as latitude increases. First, as already noticed above, at $\lambda>\lambda_{M}$, the lower-bound of $\theta_{R}$-integration actually departs from $\theta_{g}$ in equation (5), coming closer to $\theta_{r}$ to satisfy the resonance condition (2). It has been shown by Mourenas et al. [2012, see equation (B3)] that $\left(\tan \theta_{r}-\tan \theta_{R}\right) \propto$ $\Omega_{c}^{3}\left(\lambda_{M}\right) / \Omega_{c}^{3}(\lambda)$ for $\lambda \gg \lambda_{M}$. It implies a first slowing-down of the increase of diffusion rate with latitude as compared to the estimate (A1). At higher latitudes such that the upper-bound on $\theta_{R}(\lambda)$ from equation (2) becomes smaller than $\theta_{r}(\lambda)$, i.e. $\alpha_{R}(\lambda)>\pi / 2-\theta_{r}(\lambda)$, the increase with $\lambda$ of the diffusion rate abates a second time. Indeed, the $\tan \theta_{r}(\lambda)$ term at the numerator of equation (5) simply saturates there. The latitude at which it occurs is given by $\lambda_{M S}=2^{1 / 2} \sqrt{\left(\omega_{m} / \Omega_{c 0} \sin \alpha_{0}\right)^{2 / 27}-1}$. Finally, at still higher latitudes $\lambda>\lambda_{M}$ such that the upper-bound of $\theta_{R}$-integration becomes smaller than $\theta_{g}(\lambda)$, the $D^{-}$coefficient should be identically zero. In reality, some waves are still present at smaller wave-normal angles [Agapitov et al., 2011; Artemyev et al., 2012a], but their contribution to diffusion decreases as compared to the range above the Gendrin angle $\theta_{g}$ [Mourenas et al., 2012]. The corresponding limit is given by $\sin \alpha_{R}<2\left(\omega_{m}+\Delta \omega\right) / \Omega_{c}(\lambda)$. Using adiabatic invariance, it follows that

$$
\frac{\Omega_{c}\left(\lambda_{R}\right)}{\Omega_{c 0}}<\left(2 \frac{\omega_{m}+\Delta \omega}{\Omega_{c 0} \sin \alpha_{0}}\right)^{2 / 3} .
$$

[47] Expanding the dipolar magnetic field expression for small $\lambda$, one gets also $\Omega_{c}(\lambda) / \Omega_{c 0} \sim\left(1+\lambda^{2} / 2\right)^{9}$. Combined with equation (A2) and taking $\omega \sim \omega_{m}$, it provides an estimate of the maximum latitude $\lambda_{M M}$ for significant diffusion contribution:

$$
\lambda_{M M}^{2} / 2 \approx\left(\frac{2 \omega_{m}}{\Omega_{c 0} \sin \alpha_{0}}\right)^{2 / 27}-1 .
$$

[48] For $L=4$ to 6 near the loss cone edge and $\omega_{m} / \Omega_{c 0} \sim 0.35$, equation (A3) gives $\lambda_{M M} \sim 35^{\circ}<\lambda_{+}$. Let us now examine in more details the different regimes of diffusion corresponding to the different latitudinal ranges. Over the domain $\lambda_{M}<\lambda<\lambda_{M M}$, one has in general $\Omega_{c}\left(\lambda_{M M}\right) /$ $\Omega_{c}\left(\lambda_{M}\right)<2.3$ for typical parameters such that $N_{r} /|n|>1$ (for example, with $\lambda_{M} \sim 12^{\circ}$ and $\lambda_{M M}\left(\alpha_{0}\right)<29^{\circ}$ or $\lambda_{M} \sim 20^{\circ}$ and $\lambda_{M M}\left(\alpha_{0}\right)<33^{\circ}$ ). For $|n|=1$ and a dipolar magnetic field, the factor $\sim 1-\tan ^{4} \theta_{R}(\lambda) / \tan ^{4} \theta_{r}(\lambda)$ at the numerator of equation (5) varies in this domain roughly like $\left(\Omega_{c}\left(\lambda_{M}\right) / \Omega_{c}\right)^{\Lambda}$ with $\Lambda \sim 1.4$ to 2 . Consequently, the actual integrand in equation (A1) becomes $\left(\Omega_{c} / \Omega_{c 0}\right)^{\nu}$ with $\nu \sim 3|n|-7 / 9-\Lambda(n)$ smaller than in (A1), which is tantamount to a progressive slowing-down of the increase of $D^{-}(n)$ with latitude. Exponent $\Lambda$ slightly decreases for higher $|n|>1$ so that the slowing-down is then more modest. At latitudes higher than $\lambda_{M S}$, however, the $\tan \theta_{r}(\lambda)$ term at the numerator of equation (5) eventually saturates, so that $\nu \sim|n|+2 / 9-\Lambda(n) \sim 0$. The increase with latitude of the diffusion contribution then comes to an end, before it finally declines between $\lambda_{M M}$ and $\lambda_{+}$.

[49] Ultimately, the $\lambda$-integral in equation (6) can be approximated by the average value of the integrand in equation (A1) multiplied by the integration range. Although the exact variation with latitude of this integrand is quite complicated, it has just been shown above that it varies actually rather weakly from $\lambda_{M}$ through $\lambda_{M S}$ to $\lambda_{M M}$. Furthermore, as it saturates near $\lambda \sim \lambda_{M S}$, an acceptable, rough estimate of the average value of this integrand is simply $\Omega_{c}^{3|n|-7 / 9}\left(\lambda_{M S}\right)$. In fact, taking $\lambda \sim \lambda_{M S}$ in the integrand of equation (A1) is also consistent with the assumption made to derive equation (A1) that the ratio of tangents in equation (5) can be approximated by $\left(\tan \theta_{r}\right)^{2|n|-1}$, since it is equivalent to taking $\theta_{R}(\lambda) \sim \theta_{r}(\lambda)$. As concerns the $\lambda$-integration range in equation (A1), one can take $\left(\lambda_{+}-\lambda_{m}\right) \sim \lambda_{M M}$ for $\left(1-\Delta \omega / \omega_{m}\right) N_{r}\left(\omega_{m}\right)<$ $2\left(\omega_{m}+\Delta \omega\right) /\left(\Omega_{c 0} \sin \alpha_{0}\right)$, i.e. close to the loss cone edge at $\sin \alpha_{0}<\omega_{m} / \Omega_{c 0}$ for $\Delta \omega / \omega_{m} \sim 0.5$. This gives finally

$$
\left\langle D_{\alpha \alpha, \pm n}^{-} / p^{2}\right\rangle_{B} \approx \frac{B_{w}^{2}}{B_{0}^{2}} \frac{3\left(1+\omega_{m}^{2} / \Omega_{c 0}^{2}\right) \omega_{m}^{13 / 27} \Omega_{c 0}^{14 / 27} \lambda_{M M}}{8 \gamma p^{2} \varepsilon_{m 0}^{2} \cos ^{3} \alpha_{0} \sin ^{41 / 27} \alpha_{0}} .
$$

[50] The approximated diffusion coefficients in equation (A4) are independent of $n$. The total bounce-averaged pitch angle diffusion coefficient $D^{-}$is the sum of positive and negative $n$-resonances such that $|n|<N_{r}$, plus the double sum from $|n|=N_{r}$ to $+\infty$ of the other resonances. The latter lead to decreasing contributions as the $\theta$-range of integration shrinks quickly with $n$ like $1 / n^{2}$ [Mourenas et al., 2012]. The total bounce-averaged coefficient $D^{-}$is therefore roughly $4 N_{r}$ times the single- $n$ term (A4).

[51] Combining equation (A2) or (A3) with equation (2), an expression for the position of the maxima of Bessel functions $J_{|n|-1}^{2}$ at small pitch angle $\alpha_{0}<\arcsin \left(2 \omega_{m} / \Omega_{c 0}\right)$ can also be obtained:

$$
\sin \alpha_{0}(n) \approx \frac{\omega_{m} n^{3 / 4}}{\Omega_{c 0}\left(N_{r}\left(\omega_{m}\right)-\gamma \omega_{m} n /|n| \Omega_{c 0}\right)^{3 / 4}} .
$$

[52] Due to the presence of a multiplying factor $1 / \sin ^{2} \alpha_{0}$ in equation (A4), the sheer increase of the individual pitch angle diffusion coefficient $D^{-}(n)$ at small $\alpha_{0}$ exhibits at most a slight inflexion at this point. However, energy diffusion coefficients rise much more gently toward small $\alpha_{0}$, because of an additional multiplying factor $\sin ^{2} \alpha_{0}$ coming from equation (12). As a result, individual $n$-resonances energy diffusion rates may display maxima around the positions defined by equation (A5). This behavior is in good agreement with full numerical calculations. Let us finally emphasize that 


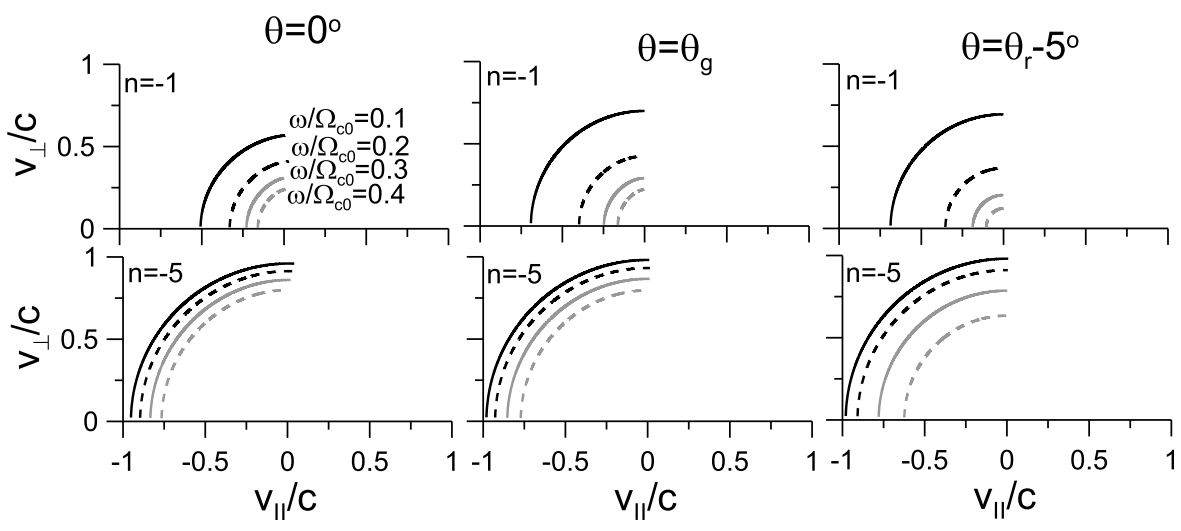

Figure B1. Resonant diffusion curves for three values of angle $\theta$ and $\Omega_{p e} / \Omega_{c}=4.5$. Initial values of ratio $\omega_{m} / \Omega_{c}$ corresponding to line $v_{\perp}=0$ are indicated in the first panel.

all the analytical estimates derived here are roughly valid only in the range

$$
2<N_{r}\left(\omega_{m}\right)<\frac{2\left(\omega_{m}+2 \Delta \omega\right)}{\sin \alpha_{0} \Omega_{c 0}} .
$$

\section{Appendix B: Resonant Diffusion Curves}

[53] In this appendix we derive expressions for the local resonant curves according to the method proposed by Summers et al. [1998]. We consider a plane wave with dimensional velocity $\beta=\omega_{m} / k c$. Although oblique wave propagation corresponds to some non-null value of the transverse velocity, we assume here for simplicity that this effect can be neglected in the frame of a first-order approximation of plane wave propagation in a transversely homogeneous system. The corresponding law of energy conservation in the system moving with the wave is $v_{\|}^{\prime}$ $d v_{\|}^{\prime}+v_{\perp}^{\prime} d v_{\perp}^{\prime}=0$, where velocity components $v_{\|}^{\prime}, v_{\perp}^{\prime}$ can be written as functions of velocity components in the rest frame:

$$
\begin{aligned}
& v_{\perp}^{\prime}=\gamma_{\phi}^{-1} \frac{\left(v_{\perp} / c\right)}{1-\beta\left(v_{\|} / c\right)} \\
& v_{\|}^{\prime}=\frac{\left(v_{\|} / c\right)-\beta}{1-\beta\left(v_{\|} / c\right)}
\end{aligned}
$$

with $\gamma_{\phi}=1 / \sqrt{1-\beta^{2}}$. Then particle energy diffusion occurs along some curve in the velocity space $\left(v_{\perp}, v_{\|}\right)$[Gendrin, 1968] described by equation

$$
\frac{d v_{\perp}^{2}}{d v_{\|}}=-2 c \frac{\beta\left(v_{\perp} / c\right)^{2}+\left(v_{\|} / c\right)-\beta}{1-\beta\left(v_{\|} / c\right)}
$$

where phase velocity $\beta$ is defined for each point in the plane $\left(v_{\perp}, v_{\|}\right)$in agreement with dispersion relation and resonance condition

$$
\left\{\begin{array}{l}
\beta n\left(1-v_{\|}^{2}-v_{\perp}^{2}\right)^{1 / 2}=\left(\omega_{m} / \Omega_{c}\right)\left(\left(v_{\|} / c\right) \cos \theta-\beta\right) \\
\left(\omega_{m} / \Omega_{c}\right)^{2}+\beta^{2}\left(\Omega_{p e} / \Omega_{c}\right)^{2}=\left(\omega_{m} / \Omega_{c}\right) \cos \theta
\end{array}\right.
$$

[54] We solve the equation for velocity components starting from the point $v_{\perp}=0$. At this point we take $\Omega_{p e} / \Omega_{c}=4.5$ and different values of $\omega_{m} / \Omega_{c}$ and calculate the initial value of $\beta$ from the dispersion relation. The initial value of $v_{\|}$is calculated from the resonant condition. Resonant curves in $\left(v_{\perp}, v_{\|}\right)$plane are shown in Figure B1 for three values of $\theta$ and for $n=-1$ and -5 . Close to the Gendrin angle or at smaller wave-normal angles, there is little difference with the parallel wave case of Summers et al. [1998]. However, at larger wave-normal angles closer to the resonance cone angle $\theta_{r}$, higher frequency waves are found to be in resonance with significantly smaller energy electrons. Note however that the present calculations are strictly valid only for small variations of $v_{\perp}$ around its initial value on the resonance curves and at a given latitude. Bounceaveraging would also be required to determine the full characteristics.

[55] Acknowledgments. Robert Lysak thanks the reviewers for their assistance in evaluating this paper.

\section{References}

Agapitov, O., V. Krasnoselskikh, Y. Zaliznyak, V. Angelopoulos, O. Le Contel, and G. Rolland (2010), Chorus source region localization in the Earth's outer magnetosphere using THEMIS measurements, Ann. Geophys., 28, 1377-1386.

Agapitov, O., V. Krasnoselskikh, Y. V. Khotyaintsev, and G. Rolland (2011), A statistical study of the propagation characteristics of whistler waves observed by Cluster, Geophys. Res. Lett., 382, L20103, doi:10.1029/2011GL049597.

Albert, J. M. (2005), Evaluation of quasi-linear diffusion coefficients for whistler mode waves in a plasma with arbitrary density ratio, J. Geophys. Res., 110, A03218, doi:10.1029/2004JA010844.

Albert, J. M. (2007), Simple approximations of quasi-linear diffusion coefficients, J. Geophys. Res., 112, A12202, doi:10.1029/2007JA012551.

Albert, J. M. (2010), Diffusion by one wave and by many waves, J. Geophys. Res., 115, A00F05, doi:10.1029/2009JA014732.

Andronov, A. A., and V. Y. Trakhtengerts (1964), Kinetic instability of the Earth's outer radiation belt, Geomagn. Aeron., 4, 233-242.

Angerami, J. J., and D. L. Carpenter (1966), Whistler studies of the plasmapause in the magnetosphere: 2. Electron density and total tube electron content near the knee in magnetospheric ionization, J. Geophys. Res., 71, 711-725

Artemyev, A., O. Agapitov, H. Breuillard, V. Krasnoselskikh, and G. Rolland (2012a), Electron pitch-angle diffusion in radiation belts: The effects of whistler wave oblique propagation, Geophys. Res. Lett., 39, L08105, doi:10.1029/2012GL051393.

Artemyev, A., O. Agapitov, V. Krasnoselskikh, H. Breuillard, and

G. Rolland (2012b), Statistical model of electron pitch angle diffusion in the outer radiation belt, J. Geophys. Res., 117, A08219, doi:10.1029/ 2012JA017826.

Barker, A. B., X. Li, and R. S. Selesnick (2005), Modeling the radiation belt electrons with radial diffusion driven by the solar wind, Space Weather, 3, S10003, doi:10.1029/2004SW000118. 
Breuillard, H., Y. Zaliznyak, V. Krasnoselskikh, O. Agapitov, A. Artemyev, and G. Rolland (2012), Chorus wave-normal statistics in the Earth's radiation belts from ray tracing technique, Ann. Geophys., 30, 1223-1233, doi:10.5194/angeo-30-1223-2012.

Bunch, N. L., M. Spasojevic, and Y. Y. Shprits (2012), Off-equatoria chorus occurrence and wave amplitude distributions as observed by the Polar Plasma Wave Instrument, J. Geophys. Res., 117, A04205, doi:10.1029/2011JA017228.

Burton, R. K., and R. E. Holzer (1974), The origin and propagation of chorus in the outer magnetosphere, J. Geophys. Res., 79, 1014-1023, doi:10.1029/JA079i007p01014.

Chum, J., and O. Santolík (2005), Propagation of whistler-mode chorus to low altitudes: Divergent ray trajectories and ground accessibility, Ann. Geophys., 23, 3727-3738, doi:10.5194/angeo-23-3727-2005.

Cully, C. M., J. W. Bonnell, and R. E. Ergun (2008), THEMIS observations of long-lived regions of large-amplitude whistler waves in the inner magnetosphere, Geophys. Res. Lett., 35, L17S16, doi:10.1029/ 2008GL033643.

Denton, R. E., K. Takahashi, I. A. Galkin, P. A. Nsumei, X. Huang, B. W. Reinisch, R. R. Anderson, M. K. Sleeper, and W. J. Hugues (2006), Distribution of density along magnetospheric field lines, J. Geophys. Res., 111, A04213, doi:10.1029/2005JA011414.

Fok, M.-C., A. Glocer, Q. Zheng, R. B. Horne, N. P. Meredith, J. M. Albert, and T. Nagai (2011), Recent developments in the radiation belt environment model, J. Atmos. Sol. Terr. Phys., 73, 1435-1443, doi:10.1016/ j.jastp.2010.09.033

Gendrin, R. (1961), Le guidage des whistlers par le champ magnetique, Planet. Space Sci., 5, 274-278, doi:10.1016/0032-0633(61)90096-4.

Gendrin, R. (1968), Pitch angle diffusion of low energy protons due to gyroresonant interaction with hydromagnetic waves, J. Atmos. Terr. Phys., 30, 1313-1330, doi:10.1016/S0021-9169(68)91158-6.

Glauert, S. A., and R. B. Horne (2005), Calculation of pitch angle and energy diffusion coefficients with the PADIE code, J. Geophys. Res., 110, A04206, doi:10.1029/2004JA010851.

Haque, N., M. Spasojevic, O. Santolík, and U. S. Inan (2010), Wave norma angles of magnetospheric chorus emissions observed on the Polar spacecraft, J. Geophys. Res., 115, A00F07, doi:10.1029/2009JA014717.

Haque, N., U. S. Inan, T. F. Bell, J. S. Pickett, J. G. Trotignon, and G. Facskó (2011), Cluster observations of whistler mode ducts and banded chorus, Geophys. Res. Lett., 38, L18107, doi:10.1029/ 2011GL049112.

Helliwell, R. A. (1965), Whistlers and Related Ionospheric Phenomena, Stanford Univ. Press, Stanford, Calif.

Horne, R. B., and R. M. Thorne (2003), Relativistic electron acceleration and precipitation during resonant interactions with whistler-mode chorus, Geophys. Res. Lett., 30(10), 1527, doi:10.1029/2003GL016973.

Horne, R. B., R. M. Thorne, S. A. Glauert, J. M. Albert, N. P. Meredith, and R. R. Anderson (2005), Timescale for radiation belt electron acceleration by whistler mode chorus waves, J. Geophys. Res., 110, A03225, doi:10.1029/2004JA010811.

Iucci, N., et al. (2005), Space weather conditions and spacecraft anomalies in different orbits, Space Weather, 3, S01001, doi:10.1029/2003SW000056.

Kellerman, A. C., and Y. Y. Shprits (2012), On the influence of solar wind conditions on the outer-electron radiation belt, J. Geophys. Res., 117 A05217, doi:10.1029/2011JA017253.

Kennel, C. F., and H. E. Petschek (1966), Limit on stably trapped particle fluxes, J. Geophys. Res., 71, 1-28.

Kersten, K., C. A. Cattell, A. Breneman, K. Goetz, P. J. Kellogg, J. R. Wygant, L. B. Wilson III, J. B. Blake, M. D. Looper, and I. Roth (2011), Observation of relativistic electron microbursts in conjunction with intense radiation belt whistler-mode waves, Geophys. Res. Lett. 38, L08107, doi:10.1029/2011GL046810.

Li, W., Y. Y. Shprits, and R. M. Thorne (2007), Dynamic evolution of energetic outer zone electrons due to wave-particle interactions during storms, J. Geophys. Res., 112, A10220, doi:10.1029/2007JA012368.

Li, W., J. Bortnik, R. M. Thorne, and V. Angelopoulos (2011), Global distribution of wave amplitudes and wave normal angles of chorus waves using THEMIS wave observations, J. Geophys. Res., 116, A12205, doi:10.1029/2011JA017035.

Lyons, L. R. (1974), Pitch angle and energy diffusion coefficients from resonant interactions with ion-cyclotron and whistler waves, J. Plasma Phys., 12, 417-432, doi:10.1017/S002237780002537X

Lyons, L. R., R. M. Thorne, and C. F. Kennel (1971), Electron pitch-angle diffusion driven by oblique whistler-mode turbulence, J. Plasma Phys., 6 , 589-606, doi:10.1017/S0022377800006310.

Lyons, L. R., R. M. Thorne, and C. F. Kennel (1972), Pitch-angle diffusion of radiation belt electrons within the plasmasphere, J. Geophys. Res., 77, 3455-3474, doi:10.1029/JA077i019p03455.
Lyons, L. R., D.-Y. Lee, R. M. Thorne, R. B. Horne, and A. J. Smith (2005), Solar wind-magnetosphere coupling leading to relativistic electron energization during high-speed streams, J. Geophys. Res., 110 A11202, doi:10.1029/2005JA011254

Meredith, N. P., R. B. Horne, and R. R. Anderson (2001), Substorm dependence of chorus amplitudes: Implications for the acceleration of electrons to relativistic energies, J. Geophys. Res., 106, 13,165-13,178, doi:10.1029/2000JA900156.

Miyoshi, Y., and R. Kataoka (2011), Solar cycle variations of outer radiation belt and its relationship to solar wind structure dependences, J. Atmos. Sol. Terr. Phys., 73, 77-87, doi:10.1016/j.jastp.2010.09.031.

Mourenas, D., and J.-F. Ripoll (2012), Analytical estimates of quasi-linear diffusion coefficients and electron lifetimes in the inner radiation belt, J. Geophys. Res., 117, A01204, doi:10.1029/2011JA016985.

Mourenas, D., A. V. Artemyev, J.-F. Ripoll, O. V. Agapitov, and V. V. Krasnoselskikh (2012), Timescales for electron quasi-linear diffusion by parallel and oblique lower-band chorus waves, J. Geophys. Res., 117 , A06234, doi:10.1029/2012JA017717.

Ni, B., R. M. Thorne, Y. Y. Shprits, and J. Bortnik (2008), Resonant scattering of plasma sheet electrons by whistler-mode chorus: Contribution to diffuse auroral precipitation, Geophys. Res. Lett., 35, L11106, doi:10.1029/2008GL034032.

Ni, B., R. M. Thorne, N. P. Meredith, Y. Y. Shprits, and R. B. Horne (2011), Diffuse auroral scattering by whistler mode chorus waves: Dependence on wave normal angle distribution, J. Geophys. Res., 116 , A10207, doi:10.1029/2011JA016517.

Nishimura, Y., et al. (2010), Identifying the driver of pulsating aurora, Science, 330, 81-84, doi:10.1126/science.1193186.

Orlova, K. G., and Y. Y. Shprits (2010), Dependence of pitch-angle scattering rates and loss timescales on the magnetic field model, Geophys. Res. Lett., 37, L05105, doi:10.1029/2009GL041639.

Pokhotelov, D., F. Lefeuvre, R. B. Horne, and N. Cornilleau-Wehrlin (2008), Survey of ELF-VLF plasma waves in outer radiation belt observed by Cluster STAFF-SA experiment, Ann. Geophys., 26, 3269-3277, doi:10.5194/angeo-26-3269-2008.

Roth, I., M. Temerin, and M. K. Hudson (1999), Resonant enhancement of relativistic electron fluxes during geomagnetically active periods, Ann. Geophys., 17, 631-638, doi:10.1007/s00585-999-0631-2.

Santolík, O., D. A. Gurnett, J. S. Pickett, J. Chum, and N. CornilleauWehrlin (2009), Oblique propagation of whistler mode waves in the chorus source region, J. Geophys. Res., 114, A00F03, doi:10.1029/ 2009JA014586.

Sheeley, B. W., M. B. Moldwin, H. K. Rassoul, and R. R. Anderson (2001), An empirical plasmasphere and trough density model: CRRES observations, J. Geophys. Res., 106, 25,631-25,642, doi:10.1029/2000JA000286.

Shklyar, D., J. Chum, and F. Jirícek (2004), Characteristic properties of $\mathrm{Nu}$ whistlers as inferred from observations and numerical modelling, Ann. Geophys., 22, 3589-3606, doi:10.5194/angeo-22-3589-2004.

Shprits, Y. Y., R. M. Thorne, R. B. Horne, and D. Summers (2006), Bounce-averaged diffusion coefficients for field-aligned chorus waves, J. Geophys. Res., 111, A10225, doi:10.1029/2006JA011725.

Shprits, Y. Y., N. P. Meredith, and R. M. Thorne (2007), Parameterization of radiation belt electron loss timescales due to interactions with chorus waves, Geophys. Res. Lett., 34, L11110, doi:10.1029/2006GL029050.

Shprits, Y. Y., D. A. Subbotin, N. P. Meredith, and S. R. Elkington (2008), Review of modeling of losses and sources of relativistic electrons in the outer radiation belt II: Local acceleration and loss, J. Atmos. Sol. Terr. Phys., 70, 1694-1713, doi:10.1016/j.jastp.2008.06.014

Shprits, Y. Y., D. Subbotin, and B. Ni (2009), Evolution of electron fluxes in the outer radiation belt computed with the VERB code, J. Geophys. Res., 114, A11209, doi:10.1029/2008JA013784.

Stix, T. H. (1962), The Theory of Plasma Waves, McGraw-Hill, New York. Summers, D., R. M. Thorne, and F. Xiao (1998), Relativistic theory of wave-particle resonant diffusion with application to electron acceleration in the magnetosphere, J. Geophys. Res., 103, 20,487-20,500, doi:10.1029/98JA01740.

Summers, D., C. Ma, N. P. Meredith, R. B. Horne, R. M. Thorne, D. Heynderickx, and R. R. Anderson (2002), Model of the energization of outer-zone electrons by whistler-mode chorus during the October 9, 1990 geomagnetic storm, Geophys. Res. Lett., 29(24), 2174, doi:10.1029/ 2002GL016039.

Summers, D., B. Ni, and N. P. Meredith (2007), Timescales for radiation belt electron acceleration and loss due to resonant wave-particle interactions: 1. Theory, J. Geophys. Res., 112, A04206, doi:10.1029/ 2006JA011801.

Tao, X., J. Bortnik, J. M. Albert, K. Liu, and R. M. Thorne (2011), Comparison of quasilinear diffusion coefficients for parallel propagating whistler mode waves with test particle simulations, Geophys. Res. Lett., 38 , L06105, doi:10.1029/2011GL046787. 
Thorne, R. M., T. P. O’Brien, Y. Y. Shprits, D. Summers, and R. B. Horne (2005), Timescale for $\mathrm{MeV}$ electron microburst loss during geomagnetic storms, J. Geophys. Res., 110, A09202, doi:10.1029/2004JA010882.

Trakhtengerts, V. Y. (1999), A generation mechanism for chorus emission, Ann. Geophys., 17, 95-100, doi:10.1007/s00585-999-0095-4.

Tsurutani, B. T., and E. J. Smith (1977), Two types of magnetospheric ELF chorus and their substorm dependences, J. Geophys. Res., 82, 5112-5128, doi:10.1029/JA082i032p05112.

Ukhorskiy, A. Y., M. I. Sitnov, R. M. Millan, and B. T. Kress (2011), The role of drift orbit bifurcations in energization and loss of electrons in the outer radiation belt, J. Geophys. Res., 116, A09208, doi:10.1029/2011JA016623. van Milligen, B. P., P. D. Bons, B. A. Carreras, and R. Sánchez (2005) On the applicability of Fick's law to diffusion in inhomogeneous systems, Eur. J. Phys., 26, 913-925, doi:10.1088/0143-0807/26/5/023.
Varotsou, A., D. Boscher, S. Bourdarie, R. B. Horne, N. P. Meredith, S. A. Glauert, and R. H. Friedel (2008), Three-dimensional test simulations of the outer radiation belt electron dynamics including electron-chorus resonant interactions, J. Geophys. Res., 113, A12212, doi:10.1029/ 2007JA012862.

Walt, M. (1994), Introduction to Geomagnetically Trapped Radiation, Cambridge Atmos. Space Sci. Ser., vol. 10, Cambridge Univ. Press, Cambridge, U. K

Xiao, F., Z. Su, H. Zheng, and S. Wang (2009), Modeling of outer radiation belt electrons by multidimensional diffusion process, J. Geophys. Res. 114, A03201, doi:10.1029/2008JA013580. 